\title{
Irreducible representations of metrizable spaces and strongly countable-dimensional spaces
}

\author{
by
}

\author{
Richard P. Mills paugh (Grand Forks, N.D.), \\ Leonard R. R u bi in (Norman, Okla.) \\ and Philip J. S c hapi r o (Langston, Okla.)
}

\begin{abstract}
We generalize Freudenthal's theory of irreducible representations of metrizable compacta by inverse sequences of compact polyhedra to the class of all metrizable spaces. Our representations consist of inverse sequences of completely metrizable polyhedra which are ANR's. They are extendable: any such representation of a closed subspace of a given metrizable space extends to another such of the entire space. We use our techniques to characterize strongly countable-dimensional metrizable spaces.

1. Introduction. It is a classical result of Freudenthal [Fr] that every metrizable compactum can be written as the limit of an inverse sequence of compact polyhedra with bonding maps which are irreducible and piecewise linear. In $[\mathrm{J}-\mathrm{R}]$ we proved a stronger version of this theorem which provides, among other things, an extendability property. The idea here is to choose, for a given $X$, an irreducible inverse sequence representation so that whenever $X$ is a closed subspace of another metrizable compactum $Y$, then $Y$ has an irreducible representation (of the same "type" as the one for $X$ ), which is an extension of the one for $X$. All the new bonding maps are extensions and the coordinate projection maps are extensions of the previous ones. Since the system for $Y$ is of the same "type" as the one for $X$, it again is extendable, and one may continue to produce such extendable extensions ad infinitum.

To provide such a theory for arbitrary metrizable spaces $X$ introduces some major difficulties. The first one involves a question of which types of polyhedra to use. If $X$ is not compact, then we could not expect it to be the limit of an inverse sequence of compact polyhedra. If $X$ is not separable, then we could not even embed it in such a limit.
\end{abstract}

1991 Mathematics Subject Classification: Primary 54B35, 54F45.

Key words and phrases: inverse sequence, irreducible representation, extendability, strong countable-dimensionality. 
We have chosen as our class of polyhedra those which are completely metrizable absolute neighborhood retracts (ANR's) and which are locally finite-dimensional. These seem natural to us. Now if $X$ happens not to be completely metrizable, then obviously $X$ cannot be homeomorphic to the limit of an inverse sequence of such polyhedra. We account for this by defining a notion of representation (see Section 6 ) which yields an embedding of $X$ onto a dense subspace of the limit and which happens to be a homeomorphism, with our construction, in case $X$ is topologically complete.

To get the property of local finite-dimensionality in our polyhedra, which is crucial for inducing irreducibility of our maps, we must have polyhedra which are locally finite-dimensional in the combinatorial sense. These polyhedra are the spaces of nerves of certain open covers of our given space. Classically one can always obtain arbitrarily fine open covers of a metrizable space whose nerves are locally finite-dimensional by directly applying theory developed by Dowker in [Do]. We, however, are faced with the problem of delicately extending such open covers from a closed subspace to those of the rest of the space. To do this we had to do more than just apply the results in [Do]; indeed, we had to analyze one of his proofs and bring to light certain extension attributes that his construction yields. This will become apparent in Section 5 below.

Previous results along these lines ([Is1], [Is2], [Ko1]) are discussed, for example, in [Ko2]. These differ in certain significant ways from the ones that will be found herein. On the one hand, our approach (but none of theirs) contains the property of extendability, which was crucial in $[\mathrm{J}-\mathrm{R}]$. On the other hand, our method involves an axiomatic, recursive type of construction, which can be used in certain applications. Indeed, it was this recursive procedure which, because of its flexibility, was decisive in [R-S] where it was shown that if $X$ is a metric space and $\operatorname{dim}_{\mathbb{Z}} X \leq n$, then $X$ is the cell-like image of a metric space $Y$ with $\operatorname{dim} Y \leq n$. We anticipate that the systematic constructions herein described will be used essentially in further applications to the theory of cohomological dimension.

Let us now outline our approach. In Section 2 we develop some theory for polyhedra with arbitrary topologies. We give in Theorem 2.7 a criterion for detecting when such a polyhedron is an ANR and in Lemma 2.9 we show a relation between the combinatorial dimension of a polyhedron and a space mapping irreducibly onto it. Section 3 contains the theory on uniformities for polyhedra which will produce metrizable topologies of the desired type. Here the main result is Proposition 3.5. Such uniformities were important in [R-S].

In Section 4 we review notions of nerves and canonical maps. The definition of an "expansion" of an open cover of a closed subspace to an open cover of the entire space is made. We give in Section 5 the definition of a Dowker system. This is a way to describe the hidden structure that exists 
in [Do] in the proof of existence of arbitrarily fine open covers with locally finite-dimensional nerves. It leads to a concept of principal refinement of a locally finite open cover of a metric space $X$. Although a Dowker system yields refinements in a canonical manner, and this is very important to our construction, the principal refinements are not at all canonical. Since we have to extend them anyway, it is necessary that we keep track of the manner in which they refine certain given covers, and this leads to much complication as we proceed with this method.

Section 6 contains the notion of a simplicially irreducible representation. Our representation involves an embedding of the given space onto a dense subspace of the limit of an inverse sequence of polyhedra. However, we make stringent requirements on the bonding maps, the polyhedra, and the representation. In case the given space has a complete metric, this embedding as done by our construction will be a homeomorphism. We also define the concept of a definitive system for a metric space. Such a definitive system always induces a simplicially irreducible representation for the space.

Our work in Section 7 involves proving that every metric space supports a definitive system and hence has a simplicially irreducible representation. We do more, though, by laying the groundwork for extendable representations. The main tool is Lemma 7.3, and the main result is Lemma 7.7. We apply the basic concepts of irreducible representations in Section 8 where we give a characterization of strong countable dimension. The key to this is showing that each open cover of our given space can be refined by a locally finite open cover whose nerve is locally finite-dimensional and whose local order at each point $x$ is not greater than the value of a certain prescribed function evaluated at $x$ (Proposition 8.8). In our final section, we give our definition of extendable and recursively extendable representations. Theorem 9.5 contains the result on existence of such representations, and, in case we are dealing with completely metrizable spaces, it states that we can make the representations (recursively) faithful.

2. Polyhedra which are ANR's. Part of our main construction involves putting a metric topology on the polyhedron of a simplicial complex by means of a countable base for a uniformity. We shall want this uniformity to be complete and the resulting space to be an ANR. Let us now present some basic theory which will help us produce the desired structures.

For our theory of uniformities we shall use the notion of uniform covers [Wi, 36, p. 244] instead of the idea of surroundings or entourages [Wi, 35, p. 238].

Let $K$ be a simplicial complex; by $|K|$ we mean, as in [Sp, 3.1], the polyhedron of $K$ with no topology implied. The $n$-skeleton of $K$ will be denoted by $K^{(n)}$. Let us use $|K|_{\mathcal{T}}$ to denote $|K|$ with a given topology $\mathcal{T}$. 
The Whitehead (weak) topology is designated $C W$. There is a metric $d$ for $|K|$, "induced by $K$ " (see the appendix of $[\mathrm{M}-\mathrm{S}]$ ), and given by

$$
d(x, y)=\sum_{v \in K^{(0)}}\left|\beta_{v}(x)-\beta_{v}(y)\right|,
$$

where $\beta_{v}$ denotes the $v$-barycentric coordinate function for a vertex $v$ of $K$. We shall use $\mathcal{T}_{d}$ to designate the topology generated by this metric $d$. It is well known that the metric

$$
d^{\prime}(x, y)=\sqrt{\sum_{v \in K^{(0)}}\left(\beta_{v}(x)-\beta_{v}(y)\right)^{2}}
$$

yields the same topology. We shall also say that $d^{\prime}$ is "induced by $K$ ".

As usual, for $v \in K^{(0)}, \operatorname{st}(v, K)$ is the open star of $v$ in $|K|$ and $\mathrm{st}^{k+1}(v, K)$ is the open star of $\operatorname{st}^{k}(v, K)$, namely, the union of the elements of $\operatorname{st}(v, K)$ which intersect $\mathrm{st}^{k}(v, K)$. Let us use $\mathrm{st}^{k} K$ for $\left\{\mathrm{st}^{k}(v, K) \mid v \in K^{(0)}\right\}$, and st $K$ for $\mathrm{st}^{1} K$. We shall treat $\overline{\mathrm{st}}(v, K)$, the closed star of $v$ in $K$, as the subcomplex of $K$ consisting of all simplexes of $K$ which are faces of simplexes of $K$ having $v$ as a vertex. If $L$ is a subdivision of $K$, then $|L|_{C W}=|K|_{C W}$, and st $L$ is an open cover of $|L|_{C W}$.

If $\mathcal{T}$ is a metrizable topology for $|K|$ and $|K|_{\mathcal{T}}$ is locally finite-dimensional, then $|K|_{\mathcal{T}}$ is an ANR if and only if $|K|_{\mathcal{T}}$ is locally contractible (see II.17.1 of $[\mathrm{Hu}])$. Of course, if $v \in K^{(0)}$, then $|\overline{\operatorname{st}}(v, K)|_{C W}$ is contractible. These facts will be useful in showing that the metrizable polyhedra employed in this work are ANR's.

2.1. Definition. Let $f, g: X \rightarrow|K|$ be functions, where $K$ is an arbitrary simplicial complex. Then we say that $g$ is a $K$-modification of $f$ if for each $x \in X$ and simplex $\tau$ of $K$, whenever $f(x) \in \tau$, then also $g(x) \in \tau$. (Equivalently, one can say that whenever $f(x)$ lies in the interior of a simplex $\tau$ of $K$, then $g(x) \in \tau$.)

2.2. Lemma. Suppose $K$ is a simplicial complex and $\mathcal{L}$ is a collection of subdivisions of $K$ having the property that $\mathcal{B}=\{$ st $L \mid L \in \mathcal{L}\}$ is a base for a uniformity $\mathcal{U}$ on $|K|$. Let $\mathcal{T}$ be the topology on $|K|$ determined by $\mathcal{U}$. Then for each $x \in|K|$ and neighborhood $V$ of $x$ in $|K|_{\mathcal{T}}$, there exists $L \in \mathcal{L}$ and $v \in L^{(0)}$ such that $x \in|\overline{\operatorname{st}}(v, L)| \subset V$.

Pr o of. Since $\mathcal{B}$ is a base for $\mathcal{U}$, there is an $M \in \mathcal{L}$ such that $\operatorname{st}(x, \operatorname{st} M)$ $\subset V$. Further, there exists $L \in \mathcal{L}$ such that st $L$ is a star-refinement of the cover st $M$. Now for some $v \in L^{(0)}, x \in \operatorname{st}(v, L)$. Hence for some $w \in L^{(0)}, x \in \operatorname{st}(\operatorname{st}(w, L)$, st $L) \subset \operatorname{st}(x$, st $M)$. Choose $v \in L^{(0)}$ so that $x \in \operatorname{st}(v, L)$ and $\operatorname{st}(v, L) \cap \operatorname{st}(w, L) \neq \emptyset$. There exists $Q \in \operatorname{st} M$ such that $x \in \operatorname{st}(\operatorname{st}(v, L)$, st $L) \subset Q$ and hence $Q \subset \operatorname{st}(x$, st $M)$. Finally, just note that $x \in \operatorname{st}(v, L) \subset|\overline{\operatorname{st}}(v, L)| \subset \operatorname{st}(\operatorname{st}(v, L)$, st $L) \subset Q \subset \operatorname{st}(x, \operatorname{st} M) \subset V$. 
Let us agree that whenever $\sigma$ is a simplex, we shall also use the symbol $\sigma$ to denote the simplicial complex consisting of $\sigma$ and all its faces.

2.3. Lemma. Let $K$ be a simplicial complex and $\mathcal{T}$ be a paracompact topology for $|K|$ such that

(i) $\mathcal{T} \subset C W$,

(ii) for each $\sigma \in K,|\sigma|_{\mathcal{T}}=|\sigma|_{C W}$, and

(iii) for each $v \in K^{(0)}, \operatorname{st}(v, K)$ is open in $|K|_{\mathcal{T}}$.

Let $i_{1}:|K|_{\mathcal{T}} \rightarrow|K|_{C W}$ be the identity function and $i_{2}:|K|_{C W} \rightarrow|K|_{\mathcal{T}}$ be the identity map. Then there is a map $j:|K|_{\mathcal{T}} \rightarrow|K|_{C W}$ which is a $K$-modification of $i_{1}$ and such that $j$ is a homotopy equivalence with homotopy inverse $i_{2}$. In fact, there is a function $H:|K| \times I \rightarrow|K|$ such that for each $t \in I, H_{t}:|K| \rightarrow|K|$ is simplex preserving and that

(iv) $H:|K|_{C W} \times I \rightarrow|K|_{C W}$ is a homotopy between $j \circ i_{2}$ and the identity on $|K|_{C W}$, and

(v) $H:|K|_{\mathcal{T}} \times I \rightarrow|K|_{\mathcal{T}}$ is a homotopy between $i_{2} \circ j$ and the identity map on $|K|_{\mathcal{T}}$.

Proof. One repeats the proof of Theorem 10 of the appendix of [M-S] with just one change: In the last paragraph of the proof (page 303), obtain the continuity of $H:|K|_{\mathcal{T}} \times I \rightarrow|K|_{\mathcal{T}}$ locally at $(x, t)$ as follows. Choose a neighborhood $G$ of $x$ such that $G \cap U_{v} \neq \emptyset$ for at most finitely many $v \in K^{(0)}$ (designated $V$ in that proof). Then $H$ carries $G \times I$ into the space of a finite subcomplex of $K$, so the continuity follows here just as it did in [M-S].

2.4. Notation. Whenever $\mathcal{U}$ is a base for a uniformity on a space $X$ and $Y \subset X$, then we shall use $\mathcal{U} \mid Y$ to designate the restriction of the base $\mathcal{U}$ to $Y$. Thus $\mathcal{U} \mid Y$ is always a base for a uniformity of $Y$ whose induced topology is the topology on $Y$ inherited from $X$, where $\mathcal{U}$ generates the topology for $X$. If $\mathcal{U}$ is a uniformity for $X$, then $\mathcal{U} \mid Y$ is also a uniformity for $Y$.

2.5. Proposition. Let $K$ be a simplicial complex and $\mathcal{L}$ a collection of subdivisions of $K$ such that $\mathcal{B}=\{$ st $L \mid L \in \mathcal{L}\}$ is a base for a uniformity $\mathcal{U}$ on $|K|$. Let $\mathcal{T}$ be the topology on $|K|$ determined by $\mathcal{U}$, and assume that $|K|_{\mathcal{T}}$ is paracompact and $\mathcal{T} \subset C W$. Suppose that for each $L \in \mathcal{L}$ and $v \in L^{(0)}, \operatorname{st}(v, L)$ is open in $|K|_{\mathcal{T}}$ and that whenever $\sigma \in K, \mathcal{U}|| \sigma \mid$ induces $C W$ on $|\sigma|$. Then $|K|_{\mathcal{T}}$ is locally contractible.

Proof. Employing Lemma 2.2, one sees that it is sufficient to show that $|\overline{\operatorname{st}}(v, L)|_{\mathcal{T}}$ is contractible for each $L \in \mathcal{L}$ and $v \in L^{(0)}$. This will be true if we can show that $|\overline{\operatorname{st}}(v, L)|_{\mathcal{T}}$ is homotopy equivalent to the contractible space $|\overline{\operatorname{st}}(v, L)|_{C W}$. One can employ Lemma 2.3 to obtain this simply by using the restriction of $H$ on $|\overline{\mathrm{st}}(v, L)| \times I$, which lands in $|\overline{\mathrm{st}}(v, L)|$ because of the simplex preserving property of each $H_{t}$. 
The next result gives a condition under which the combinatorial dimension of a simplicial complex also equals the topological dimension of its polyhedron with a given topology.

2.6. Lemma. Let $K$ be a simplicial complex, and $\mathcal{T}$ be a topology for $|K|$ such that

(i) $|K|_{\mathcal{T}}$ is a normal space,

(ii) $|\sigma|_{\mathcal{T}}=|\sigma|_{C W}$ for each $\sigma \in K$, and

(iii) whenever $L$ is a subcomplex of $K$, then $|L|$ is closed in $|K|_{\mathcal{T}}$.

Then for each $n, \operatorname{dim}\left|K^{(n)}\right|_{\mathcal{T}} \leq n$.

Proof. Let $A \subset\left|K^{(n)}\right|=\left|K^{(n)}\right|_{\mathcal{T}}$ be closed and $f: A \rightarrow S^{n}$ be a map. Suppose inductively that we have extended $f$ to a map $g: A \cup\left|K^{(r)}\right| \rightarrow S^{n}$ where $-1 \leq r<n$. We shall show that $g$ extends to a map $\psi$ of $A \cup\left|K^{(r+1)}\right|$ to $S^{n}$. Since by (iii), $\left|K^{(r+1)}\right|$ is closed in $\left|K^{(n)}\right|$, then our proof will be complete by induction.

From (iii), $A \cup\left|K^{(r)}\right|$ is closed in $\left|K^{(r+1)}\right|$, so there exists a closed neighborhood $B$ of $A \cup\left|K^{(r)}\right|$ in $\left|K^{(r+1)}\right|$ and a map $h: B \rightarrow S^{n}$ which is an extension of $g$. For each $\sigma \in K^{(r+1)}$, choose a map $\psi_{\sigma}:|\sigma| \rightarrow S^{n}$ so that $\psi_{\sigma}|| \sigma|\cap B=h||\sigma| \cap B$. Here we make use of (ii). Define the function $\widetilde{\psi}: B \cup\left|K^{(r+1)}\right| \rightarrow S^{n}$ so that $\widetilde{\psi}|B=h| B$ and $\widetilde{\psi}|| \sigma \mid=\psi_{\sigma}$ for each $\sigma \in K^{(r+1)}$. Certainly $\widetilde{\psi}$ is a well-defined function which is an extension of $g$. Once we have proved the continuity of $\widetilde{\psi}$ restricted to $A \cup\left|K^{(r+1)}\right|$, the desired map $\psi$ is $\widetilde{\psi}|A \cup| K^{(r+1)} \mid$.

If $x \in A \cup\left|K^{(r)}\right|$, then $\widetilde{\psi}$ agrees with $h$ in a neighborhood of $x$, so $\widetilde{\psi}$ is continuous at $x$. Any other $x \in A \cup\left|K^{(r+1)}\right|$ must be in the interior of an $(r+1)$-simplex $\sigma$ of $K$. Using (iii), find a neighborhood $U$ of $x$ in $A \cup\left|K^{(r+1)}\right|$ so that $U \cap A=\emptyset$ and $U \subset \operatorname{int}(\sigma)$. Then $\widetilde{\psi}=\psi_{\sigma}$ on $U$ and hence $\widetilde{\psi}$ is continuous at $x$. This completes our proof.

2.7. TheOrem. Let $K$ be a locally finite-dimensional simplicial complex, and suppose $K=K_{1}, K_{2}, \ldots$ is a sequence of subdivisions of $K$ such that $\mathcal{B}=\left\{\operatorname{st} K_{i} \mid i \in \mathbb{N}\right\}$ is a base for a metrizable uniformity $\mathcal{U}$ on $|K|$ with induced topology $\mathcal{T} \subset C W$. Suppose that for each $i \in \mathbb{N}$ and $v \in K_{i}^{(0)}$, $\operatorname{st}\left(v, K_{i}\right)$ is open in $|K|_{\mathcal{T}}$ and that whenever $\sigma \in K, \mathcal{U}|| \sigma \mid$ induces $C W$ on $|\sigma|$. Assume further that for each subcomplex $L$ of $K,|L|$ is closed in $|K|_{\mathcal{T}}$. Then $|K|_{\mathcal{T}}$ is an $A N R$.

Pro of. Certainly the conditions (i)-(iii) of Lemma 2.6 prevail, so $|K|_{\mathcal{T}}$ is locally finite-dimensional. Since $|K|_{\mathcal{T}}$ is metrizable it is paracompact, so by Proposition $2.5,|K|_{\mathcal{T}}$ is locally contractible. Therefore $|K|_{\mathcal{T}}$ is a locally finite-dimensional, locally contractible metrizable space, so it is an ANR. 
Next we shall obtain a relation between the dimension of a given space and the combinatorial dimension of a complex $K$ under certain mapping conditions.

2.8. Definition. Let $K$ be a simplicial complex, $\mathcal{T}$ be a topology for $|K|$, and $f: X \rightarrow|K|_{\mathcal{T}}$ be a map. We say that $f$ is $K$-irreducible if for each map $g: X \rightarrow|K|_{\mathcal{T}}$ such that $g$ is a $K$-modification of $f$, we have $g(X)=|K|_{\mathcal{T}}$. Since $f$ is a $K$-modification of itself, $K$-irreducible maps are always surjective.

2.9. Lemma. Let $X$ be a normal space, $K$ be a locally finite-dimensional simplicial complex, and suppose that $\mathcal{T}$ is a topology for $|K|$ such that $\mathcal{T}$ induces $C W$ on each simplex of $K$ and such that for each subcomplex $L$ of $K,|L|$ is closed in $|K|_{\mathcal{T}}$. If $f: X \rightarrow|K|_{\mathcal{T}}$ is a $K$-irreducible map, then the combinatorial dimension of $K$ is $\leq \operatorname{dim} X$.

Proof. Suppose the contrary, that there is a simplex $\sigma$ of $K$ with $\operatorname{dim} \sigma>\operatorname{dim} X$. We may as well assume that $\sigma$ is principal. Let $A=$ $|K| \backslash \operatorname{int} \sigma$. According to the hypothesis, both $A$ and $\sigma$ are closed in $|K|_{\mathcal{T}}$. Now $\operatorname{dim} f^{-1}(\sigma) \leq \operatorname{dim} X<\operatorname{dim} \sigma$. Hence there is a map $g: f^{-1}(\sigma) \rightarrow$ $|\partial \sigma|_{\mathcal{T}}$ so that $g$ equals $f$ on $f^{-1}(|\partial \sigma|)$. Take $h: X \rightarrow|K|_{\mathcal{T}}$ to be the function which equals $f$ on $f^{-1}(A)$ and $g$ on $f^{-1}(\sigma)$. Then $h$ is a map which is a $K$-modification of $f$ but is not a surjection. This contradicts the $K$-irreducibility of $f$, and completes our proof.

3. Uniformities for polyhedra. Let $K$ be a locally finite-dimensional simplicial complex. We are going to describe a kind of uniformity for the polyhedron $|K|$ which will determine a topology $\mathcal{T}$ for $|K|$ so that $|K|_{\mathcal{T}}$ is a metrizable, locally finite-dimensional ANR.

For any simplicial complex $K$, let us use $\beta_{1} K=\beta K$ to denote the barycentric subdivision of $K$ and $\beta_{n+1} K=\beta\left(\beta_{n} K\right)$ to designate the $(n+1)$ iterated barycentric subdivision of $K$.

Whenever $L$ is a subdivision of $K$, then st $L$ refines st $K$. If we let $\mathcal{V}=\operatorname{st}\left(\beta_{2} K\right)$, then it is also true that $\mathcal{V}$ is a star-refinement of st $K$. Hence we have

3.1. Lemma. If $K$ is a simplicial complex and $L$ is a subdivision of $\beta_{2} K$, then st $L$ is a star-refinement of st $K$.

3.2. Definition. Let $K=K_{0}, K_{1}, \ldots$ be a sequence of subdivisions of a simplicial complex $K$ such that for each $i \geq 0, K_{i+1}$ is a subdivision of $\beta_{2} K_{i}$. Applying Lemma 3.1, we see that $\left\{\mathcal{V}_{i}\right\}_{i=0}^{\infty}$ with $\mathcal{V}_{i}=$ st $K_{i}$ is a countable base for a uniformity $\mathcal{B}$ on $|K|$ having the property that $\mathcal{V}_{i+1}$ star-refines $\mathcal{V}_{i}$ for each $i$. We shall refer to $\mathcal{B}$ as the sequential star-uniformity for $|K|$ determined by $\left\{K_{i}\right\}_{i=0}^{\infty}$. 
Before we get to the next proposition, let us state and prove technical lemmas. The metric $d^{\prime}$ on a polyhedron $|K|$, induced by $K$, yields a "linear" metric on each simplex of $K$. We mean by this that for each $n \in \mathbb{N}$, there is a fixed simplex $\sigma_{n} \subset \mathbb{R}^{n+1}$ such that if $\sigma$ is an $n$-simplex of $K$, then for any simplicial isomorphism $\varrho: \sigma \rightarrow \sigma_{n}$, the metric $d^{\prime}$ on $\sigma$ agrees with the Euclidean metric on $\sigma_{n}$ "pulled back" by $\varrho$. We deduce from 3.3 .12 of [Sp] that when the $n$-simplex $\sigma$ has such a metric, then for any $\sigma^{\prime} \in \beta \sigma$, $\operatorname{diam} \sigma^{\prime} \leq n(n+1)^{-1} \operatorname{diam} \sigma$. Hence we have

3.3. Lemma. Let $K$ be a finite-dimensional simplicial complex and $\left\{K_{i}\right\}_{i=0}^{\infty}$ be a sequence of subdivisions of $K=K_{0}$ such that for each $i$, $K_{i+1}$ subdivides $\beta K_{i}$. Then with respect to the metric $d^{\prime}, \lim _{i \rightarrow \infty}$ mesh $K_{i}$ $=0$.

3.4. Lemma. Suppose $\beta$ is a sequential star-uniformity for $|K|$ determined by $\left\{K_{i}\right\}_{i=0}^{\infty}$, where $K=K_{0}$ is a locally finite-dimensional simplicial complex. Let $F$ be a subset of $|K|$ such that $F$ is closed in $|K|_{\mathcal{T}_{d}}$ and let $x \in|K| \backslash F$. Then for each $k \in \mathbb{N}$, there exists $i \in \mathbb{N}$ such that $\mathrm{st}^{k}\left(x\right.$, st $\left.K_{i}\right) \cap F=\emptyset$.

Proof. Let $v$ be a vertex of $K \operatorname{such}$ that $x \in \operatorname{st}(v, K)$. Then $\operatorname{st}(v, K)$ is open in $|K|_{\mathcal{T}_{d}}$ and $\overline{\operatorname{st}}(v, K)$ is finite-dimensional. Hence $|\overline{\operatorname{st}}(v, K)| \backslash F$ is a neighborhood of $x$ in $|K|_{\mathcal{T}_{d}}$. By virtue of Lemma 3.3, one may find $i \in \mathbb{N}$ such that $\operatorname{st}^{k}\left(x\right.$, st $\left.K_{i}\right) \subset \operatorname{st}(v, K) \backslash F$.

3.5. Proposition. Let $K$ be a locally finite-dimensional simplicial complex with a sequence $K=K_{0}, K_{1}, \ldots$ of subdivisions as in Definition 3.2. Let $\mathcal{B}$ be the sequential star-uniformity for $|K|$ determined by $\left\{K_{i}\right\}_{i=0}^{\infty}$ and let $\mathcal{T}$ be the topology on $|K|$ induced by $\mathcal{B}$. Then

(i) for each $i \geq 0$, st $K_{i}$ is an open cover of $|K|_{\mathcal{T}}$;

(ii) $\mathcal{T}_{d} \subset \mathcal{T} \subset C W$;

(iii) for each $\sigma \in K, \mathcal{B} \mid \sigma$ induces the topology $C W$;

(iv) $\mathcal{T}$ is Hausdorff and hence is metrizable;

(v) for each subcomplex $L$ of $K,|L|$ is closed in $|K|_{\mathcal{T}}$;

(vi) $|K|_{\mathcal{T}}$ is an $A N R$;

(vii) $\mathcal{B}$ is a complete uniformity;

(viii) $\operatorname{dim}|K|_{\mathcal{T}}$ equals the combinatorial dimension of $K$.

Proof. (i) Let $v$ be a vertex of $K_{i}$ and let $x \in \operatorname{st}\left(v, K_{i}\right)$. From Theorem 36.6 of [Wi], we only need to find $j$ so that $\operatorname{st}\left(x, \operatorname{st} K_{j}\right) \subset \operatorname{st}\left(v, K_{i}\right)$. Let $m=\operatorname{dim} \overline{\operatorname{st}}\left(v, K_{i}\right)<\infty$; let $F=\left|\operatorname{lk}\left(v, K_{i}\right)\right|$ (here, $\operatorname{lk}\left(v, K_{i}\right)$ is the link of $v$ in the complex $\left.K_{i}\right)$. By Lemma 3.4, one can find $j$ such that st $\left(x, \operatorname{st} K_{j}\right) \cap F=\emptyset$.

On the other hand, st $\left(x\right.$, st $\left.K_{j}\right)$ is connected in $\left|K_{i}\right|_{C W}$ and $F$ separates $x$ from the complement of $\overline{\operatorname{st}}\left(v, K_{i}\right)$ in $\left|K_{i}\right|_{C W}$. Hence $\operatorname{st}\left(x, \operatorname{st} K_{j}\right) \subset \operatorname{st}\left(v, K_{i}\right)$, and our proof of (i) is complete. 
(ii) Each st $K_{i}$ is an open collection in $|K|_{C W}$. From (i) and Theorem 36.6 of [Wi], it follows that $\bigcup_{i=1}^{\infty}$ st $K_{i}$ is a base for the topology $\mathcal{T}$. Hence $\mathcal{T} \subset C W$. The inclusion $\mathcal{T}_{d} \subset \mathcal{T}$ follows easily from (i) and Lemma 3.4.

(iii) From (ii) we see that the induced topology is contained in $C W$. Since the topology $C W$ is that induced by the metric $d$, Lemma 3.3 shows that $C W$ is contained in the induced topology.

(iv) Let $x$ and $y$ be distinct elements of $|K|$. Using (i), find vertices $v_{x}$ and $v_{y}$ of $K$ such that $x \in \operatorname{st}\left(v_{x}, K\right), y \in \operatorname{st}\left(v_{y}, K\right)$. Thus $\operatorname{st}\left(v_{x}, K\right) \cup \operatorname{st}\left(v_{y}, K\right)$ is a neighborhood of $\{x, y\}$ in $|K|_{\mathcal{T}}$. One sees that the finite-dimensional subcomplex $M=\overline{\operatorname{st}}\left(v_{x}, K\right) \cup \overline{\operatorname{st}}\left(v_{y}, K\right)$ of $K$ has the property that $|M|$ is a neighborhood of $\{x, y\}$ in $|K|_{\mathcal{T}}$. It is therefore sufficient to prove that $x, y$ have disjoint neighborhoods in $|M|$, and for this we will use the finitedimensionality of $M$.

Applying Lemma 3.4 with $F=\{y\}$ and $K$ replaced by $M$, find $i$ such that $\operatorname{st}\left(x, \operatorname{st} K_{i}\right) \subset \operatorname{st}\left(v_{x}, K\right)$, st $\left(y, \operatorname{st} K_{i}\right) \subset \operatorname{st}\left(v_{y}, K\right)$ and $\operatorname{st}^{3}\left(x, \operatorname{st} K_{i}\right) \cap\{y\}=\emptyset$. We then see that st $\left(x\right.$, st $\left.K_{i}\right)$ and $\operatorname{st}\left(y\right.$, st $\left.K_{i}\right)$ are disjoint neighborhoods of $x$ and $y$ in $|M|$, as required.

(v) For each subcomplex $L$ of $K,|L|$ is closed in $|K|_{\mathcal{T}_{d}}$. Therefore an application of Lemma 3.4 and (i) shows that $|L|$ is closed in $|K|_{\mathcal{T}}$.

(vi) Items (i)-(v), along with Theorem 2.7, show that $|K|_{\mathcal{T}}$ is an ANR.

(vii) Let $\mathcal{F}$ be a Cauchy filter on $|K|_{\mathcal{T}}$. It is sufficient to show that there is a point $x \in|K|$ such that the filter of $\mathcal{T}$-neighborhoods of $x$ belongs to $\mathcal{F}$.

For each $i \geq 0$, there exists $v_{i} \in K_{i}$ such that $\operatorname{st}\left(v_{i}, K_{i}\right)$ belongs to $\mathcal{F}$. If $i \leq j$, then since $\operatorname{st}\left(v_{i}, K_{i}\right) \cap \operatorname{st}\left(v_{j}, K_{j}\right) \neq \emptyset$, the vertex $v_{j}$ must lie in $\overline{\operatorname{st}}\left(v_{i}, K_{i}\right)$. Hence all $v_{i}$ belong to the finite-dimensional subcomplex $\overline{\mathrm{st}}\left(v_{0}, K\right)$.

We claim that $\left\{v_{i}\right\}_{i=0}^{\infty}$ is a Cauchy sequence. Suppose $j>0$; then we need to show that there is $k>0$ such that if $p, q \geq k$, then $v_{p}, v_{q}$ belong to $\operatorname{st}\left(v, K_{j}\right)$ for some $v \in K_{j}^{(0)}$.

There exists $A \in$ st $K_{j}$ such that st(st $\left(v_{j+1}, K_{j+1}\right)$, st $\left.K_{j+1}\right) \subset A$ since st $K_{j+1}$ is a star-refinement of st $K_{j}$. Suppose $p, q \geq j+1$. Then $\operatorname{st}\left(v_{p}, K_{p}\right) \cap$ $\operatorname{st}\left(v_{j+1}, K_{j+1}\right) \neq \emptyset$. Surely st $\left(v_{p}, K_{p}\right)$ is contained in some element of st $K_{j+1}$, and so we have $v_{p} \in \operatorname{st}\left(v_{p}, K_{p}\right) \subset A$. Similarly $v_{q} \in A$, and so $v_{p}, v_{q} \in A=$ $\operatorname{st}\left(v, K_{j}\right)$ for some $v \in K_{j}^{(0)}$.

Now two such vertices $v_{p}$ and $v_{q}$ in the Cauchy sequence cannot belong to two different open simplexes of $\overline{\operatorname{st}}\left(v_{0}, K\right)$ of the same dimension. The reader may obtain this from the following two basic facts.

FACT 1. Let $M$ be a subdivision of $\beta_{2} K$. Suppose $\sigma$ is a simplex of $K$ and $w$ is a vertex of $M$ lying in int $\sigma$. Then there exists a vertex $v$ of $\beta_{2} K$ lying in int $\sigma$ and such that $\operatorname{st}(w, M) \subset \operatorname{st}\left(v, \beta_{2} K\right)$. 
FACT 2. Let $\sigma, \tau$ be simplexes of $K$ such that neither is a face of the other. Let $v, w$ be vertices of $\beta_{2} K$ with $v \in \operatorname{int} \sigma, w \in \operatorname{int} \tau$. Then $\operatorname{st}\left(v, \beta_{2} K\right)$ $\cap \operatorname{st}\left(w, \beta_{2} K\right)=\emptyset$.

The dimension of $\overline{\operatorname{st}}\left(v_{0}, K\right)$ being finite, there must be a simplex $\sigma$ of $\overline{\operatorname{st}}\left(v_{0}, K\right)$ such that $v_{i} \in \sigma$ for all $i$. Using (iii), one sees that the Cauchy sequence $\left\{v_{i}\right\}_{i=0}^{\infty}$ in $\sigma$ converges to a point $x \in \sigma$. We complete the proof by showing that $x$ has a neighborhood base belonging to $\mathcal{F}$.

Let $j \in \mathbb{N}$. There is a $k \geq j+1$ such that $v_{k} \in \operatorname{st}\left(x, \operatorname{st} K_{j+1}\right)$. Choose $A \in \operatorname{st} K_{j+1}$ so that $v_{k}, x \in A$. Note that $v_{k} \in \overline{\operatorname{st}}\left(v_{j+1}, K_{j+1}\right)$. So $x \in$ st $\left(\operatorname{st}\left(v_{j+1}, K_{j+1}\right)\right.$, st $\left.K_{j+1}\right)$, and this latter is contained in an element $B$ of st $K_{j}$. We then have $B \subset \operatorname{st}\left(x, K_{j}\right)$, leading to $\operatorname{st}\left(v_{j+1}, K_{j+1}\right) \subset B \subset$ $\operatorname{st}\left(x, K_{j}\right)$. We already know that $\operatorname{st}\left(v_{j+1}, K_{j+1}\right)$ is in $\mathcal{F}$, and hence $\operatorname{st}\left(x, K_{j}\right)$ $\in \mathcal{F}$. Our proof of (vii) is complete.

(viii) This comes from (iv), (iii), (v) and Lemma 2.6.

3.6. Corollary. Under the hypotheses of Proposition 3.5, the barycentric coordinates $\beta_{v}:|K|_{\mathcal{T}} \rightarrow[0,1]\left(v \in K^{(0)}\right)$ are continuous.

Proof. This follows from the fact that $\mathcal{T}_{d} \subset \mathcal{T}$ and that $\beta_{v}:|K|_{\mathcal{T}_{d}} \rightarrow$ $[0,1]$ is continuous for all $v \in K^{(0)}$ (see, e.g., the appendix of [M-S]).

The next result can be proved by applying Theorem 4 of Appendix 1 of [M-S]. We leave this to the reader.

3.7. TheOREM. Let $L$ be a subcomplex of a simplicial complex $K$. Let $\mathcal{U}$ be an open cover of $|K|_{C W}$ such that $\overline{\mathrm{st}} L$ refines $\mathcal{U}|| L \mid$. Then there exists a subdivision $\widetilde{K}$ of $K$ relative to $L$ (i.e., $L$ is a subcomplex of $\widetilde{K}$ ) such that $\overline{\text { st }} \widetilde{K}$ refines $\mathcal{U}$.

4. Nerves and canonical maps. Let $\mathcal{U}$ be a collection of subsets of a space $X$. Recall that the nerve $N(\mathcal{U})$ of $\mathcal{U}$ is the simplicial complex whose vertices are the non-empty elements of $\mathcal{U}$ and such that a finite subset $\mathcal{F}$ of $\mathcal{U}$ determines a simplex of $N(\mathcal{U})$ if and only if $\bigcap \mathcal{F} \neq \emptyset$. If $\mathcal{V}$ is another collection of subsets of $X$ and $\mathcal{V}$ refines $\mathcal{U}$, then a projection of $\mathcal{V}$ in $\mathcal{U}$ is a function $\lambda: \mathcal{V} \rightarrow \mathcal{U}$ such that for each $V \in \mathcal{V}, V \subset \lambda(V)$. If $\lambda$ is a projection, then $f$ uniquely determines a simplicial map $\varphi: N(\mathcal{V}) \rightarrow N(\mathcal{U})$ whose vertex map is $\lambda$.

There is an "indexed" version of these concepts. Suppose that $\mathcal{U}=$ $\left\{U_{\gamma} \mid \gamma \in \Gamma\right\}$ is an indexed collection of subsets of a space $X$. Then its (indexed) nerve $N(\mathcal{U})$ is the simplicial complex whose vertices are the elements $\gamma$ of $\Gamma$ such that $U_{\gamma} \neq \emptyset$ and such that a finite subset $\mathcal{F}$ of $\Gamma$ determines a simplex of $N(\mathcal{U})$ if and only if $\bigcap\left\{U_{\gamma} \mid \gamma \in \mathcal{F}\right\} \neq \emptyset$. In the sequel, all notions about nerves (e.g., canonical maps) have respective counterparts in 
an indexed version. We shall not explicitly make all the definitions, but shall use them when needed, leaving it to the reader to fill in details.

Let us now state some results which occur in [M-U].

4.1. Lemma (Lemma 4 of [M-U]). Let $f: X \rightarrow \sigma$ be a $\sigma$-irreducible map of a normal space $X$ to a simplex $\sigma$. If $\tau$ is a proper face of $\sigma$, then the restriction $f \mid f^{-1}(\tau): f^{-1}(\tau) \rightarrow \tau$ is $\tau$-irreducible.

4.2. Lemma (Lemma 6 of [M-U]). Let $\mathcal{V}$ be a normal open cover of a topological space $X$. Then there exists a locally finite open cover $\mathcal{U}$ of $X$ which refines $\mathcal{V}$ and has the property that $N(\mathcal{V})$ is locally finite-dimensional.

4.3. Theorem (Theorem 2 of $[\mathrm{M}-\mathrm{U}])$. Let $f:(X, A) \rightarrow\left(|K|_{C W},|M|_{C W}\right)$ be a map of pairs, where $X$ is a normal space, $A$ is a subspace of $X, K$ is a simplicial complex and $M$ is a full subcomplex of $K$. If the restriction $\left.f|A: A \rightarrow| M\right|_{C W}$ is $M$-irreducible, then there exists a subcomplex $L$ of $X, M \subset L$, and a K-modification $g: X \rightarrow|L|_{C W}$ of $f$ such that $g$ is $L$-irreducible and $g \mid A=f$.

We have included Lemma 4.2, which could also have been derived from [Do], only for the sake of completeness. This lemma is not adequate for our purposes. The approach needed for our work, in the class of metric spaces, will come to light in Section 5.

Let $K$ be a simplicial complex, $\mathcal{T}$ be a topology for $|K|$, and $f: X \rightarrow$ $|K|_{\mathcal{T}}$ be a map. Suppose that $K$ is the nerve of an open cover $\mathcal{U}$ of $X$. Then one defines $f$ to be $\mathcal{U}$-canonical if $f^{-1} \operatorname{st}(U, K) \subset U$ for each $U \in \mathcal{U}$, $\mathcal{U}$-barycentric if $f^{-1} \operatorname{st}(U, K)=U$ for each $U \in \mathcal{U}$, and $\mathcal{U}$-normal if $f$ is both $\mathcal{U}$-barycentric and $K$-irreducible. In the latter case we say that $\mathcal{U}$ is a strongly normal cover of $X$.

4.4. Lemma. Let $\mathcal{V}$ be a locally finite open cover of a normal space $X$, assume that $N=N(\mathcal{V})$ is locally finite-dimensional, and suppose $f: X \rightarrow$ $|N|_{C W}$ is a $\mathcal{V}$-normal map. Let $\mathcal{T}$ be a topology for $|N|$ such that $\mathcal{T} \subset C W$ and $\mathcal{T}$ induces $C W$ on each simplex of $N$. Then $f: X \rightarrow|N|_{\mathcal{T}}$ is a $\mathcal{V}$-normal map.

Proof. The continuity of $f: X \rightarrow|N|_{\mathcal{T}}$ is assured by the fact that $\mathcal{T} \subset C W$. Further, $f^{-1} \operatorname{st}(U, N)=U$ is true, so that $f: X \rightarrow|N|_{\mathcal{T}}$ is a $\mathcal{U}$-barycentric map. To show that it is $N$-irreducible, suppose that $g: X \rightarrow$ $|N|_{\mathcal{T}}$ is an $N$-modification of $f$ which is not onto. Then there is a simplex $\tau$ of $N$ having a point $x$ in int $\tau$ such that $x$ is not in the image of $g$. Since $g$ is an $N$-modification of $f$, it carries $f^{-1}(\tau)$ into $\tau$. Hence one sees that $f \mid f^{-1}(\tau): f^{-1}(\tau) \rightarrow \tau$ is not an essential map and so is not $\tau$-irreducible.

Since $N$ is locally finite-dimensional, there exists a principal simplex $\sigma$ of $N$ such that $\tau$ is a face of $\sigma$. We see, as above for $\tau$, that $g$ carries $f^{-1}(\sigma)$ into $\sigma$. Now the map $f \mid f^{-1}(\sigma): f^{-1}(\sigma) \rightarrow \sigma$ must be an essential map or 
else there would be an $N$-modification $h: X \rightarrow|N|_{C W}$ of $f$ which agrees with $f$ outside $f^{-1}(\sigma)$ and whose image contains no points of int $\sigma$. This is impossible since $f: X \rightarrow|N|_{C W}$ is $\mathcal{V}$-normal and hence is $N$-irreducible.

Thus $f \mid f^{-1}(\sigma): f^{-1}(\sigma) \rightarrow \sigma$ is $\sigma$-irreducible. According to Lemma 4.1, the map $f \mid f^{-1}(\tau): f^{-1}(\tau) \rightarrow \tau$ must be $\tau$-irreducible. This is a contradiction to a previous statement about this map. Our proof is complete.

4.5. Lemma. Let $X$ be a space, $K$ be a simplicial complex, $\mathcal{T}$ be a topology for $|K|$ such that open stars of vertices are open in $|K|_{\mathcal{T}}$, and $f: X \rightarrow|K|_{\mathcal{T}}$ be a $K$-irreducible map. Then the function $\theta: K^{(0)} \rightarrow f^{-1}($ st $K)$ given by $\theta(v)=f^{-1} \operatorname{st}(v, K)$ induces an isomorphism of $K$ onto the nerve of $f^{-1}(\mathrm{st} K)$. Using this relation to identify $K$ with the nerve of $f^{-1}(\mathrm{st} K)$, we deduce that $f$ is an $f^{-1}($ st $K)$-normal map.

Proof. The $K$-irreducible map $f$ is surjective. Whenever $v, w \in K^{(0)}$ and $v \neq w$, then $\operatorname{st}(v, K) \neq \operatorname{st}(w, K)$. Hence $f^{-1} \operatorname{st}(v, K) \neq f^{-1} \operatorname{st}(w, K)$, so $\theta$ is injective. If $v_{0}, \ldots, v_{m}$ are the vertices of a simplex $\sigma$ of $K$, then $\bigcap_{i=0}^{m} \operatorname{st}\left(v_{i}, K\right) \neq \emptyset$, and so $\bigcap_{i=0}^{m} f^{-1} \operatorname{st}\left(v_{i}, K\right) \neq \emptyset$. This shows that $\theta$ is simplicial. On the other hand, if $\bigcap_{i=0}^{m} f^{-1} \operatorname{st}\left(v_{i}, K\right) \neq \emptyset$, where each $v_{i} \in K^{(0)}$, then $\bigcap_{i=0}^{m} \operatorname{st}\left(v_{i}, K\right) \neq \emptyset$ so that $v_{0}, \ldots, v_{m}$ are the vertices of a simplex of $K$. This shows that $\theta$ is surjective.

We leave to the reader to see how this shows that $f$ identifies with an $f^{-1}$ (st $K$ )-barycentric map which is $K$-irreducible, where $K$ is the nerve of $f^{-1}($ st $K)$ as previously indicated.

4.6. Lemma. Let $X$ be a closed subspace of a normal space $Y$, let $L$ be a subcomplex of a simplicial complex $K$, and let $g:(Y, X) \rightarrow\left(|K|_{C W},|L|_{C W}\right)$ be a map of pairs. Assume that for each $y \in Y$, there exists a finite subcomplex $K_{y}$ of $K$ and a neighborhood $U_{y}$ of $y$ in $Y$ such that $g\left(U_{y}\right) \subset\left|K_{y}\right|_{C W}$. Suppose that $f: X \rightarrow|L|_{C W} \subset|K|_{C W}$ is a map which is a K-modification of $\left.g|X: X \rightarrow| L\right|_{C W}$. Then there exists a map $F: Y \rightarrow|K|_{C W}$ such that $F$ is a $K$-modification of $g$ and $\left.F|X=f: X \rightarrow| L\right|_{C W}$.

Proof. For each $i=0,1, \ldots$, let $Y_{i}=g^{-1}\left(\left|K^{(i)}\right|\right)$. Fix $v \in K^{(0)}$. On $g^{-1}(v) \cap X$ the maps $g$ and $f$ must agree since $f$ is a $K$-modification of $g$. Let us define $G_{0, v}: g^{-1}(v) \rightarrow|K|_{C W}$ to equal $g$ on that set. Then define $F_{0}: Y_{0} \rightarrow|K|_{C W}$ to be $\bigcup\left\{G_{0, v} \mid v \in K^{(0)}\right\}$.

Suppose we have defined maps $F_{i}: Y_{i} \rightarrow|K|_{C W}, i=0,1, \ldots, n$ in such a manner that $F_{i}\left|Y_{i} \cap X=f\right| Y_{i} \cap X: Y_{i} \cap X \rightarrow|L|_{C W}$ and $F_{i}$ is a $K$ modification of $g \mid Y_{i}$ for each $i \leq n$, and so that $\left.F_{i+1}\left|Y_{i}=F_{i}: Y_{i} \rightarrow\right| K\right|_{C W}$ for each $i<n$.

To obtain $F_{n+1}$, first fix $\sigma \in K^{(n+1)} \backslash K^{(n)}$. Consider the two closed subsets $g^{-1}(\sigma) \cap Y_{n}$ and $g^{-1}(\sigma) \cap X$ of $g^{-1}(\sigma)$, letting $Q_{\sigma}$ denote their union. Define $G_{\sigma}: Q_{\sigma} \rightarrow|K|_{C W}$ to equal $F_{n}$ on $g^{-1}(\sigma) \cap Y_{n}=g^{-1}(\partial \sigma)$ and to 
equal $f$ on $g^{-1}(\sigma) \cap X$. Then $G_{\sigma}$ is well defined and continuous on $Q_{\sigma}$. Since $F_{n}$ is a $K$-modification of $g$ on $g^{-1}(\partial \sigma)$ and $f$ is a $K$-modification of $g$ on $g^{-1}(\sigma) \cap X$, it follows that $G_{\sigma}$ is a $K$-modification of $g$ on $Q_{\sigma}$; in fact, $G_{\sigma}\left(Q_{\sigma}\right) \subset \sigma$. Since $\sigma$ is a compact absolute retract, there exists an extension $G_{\sigma}^{\prime}$ of $G_{\sigma}$ to a map $G_{\sigma}^{\prime}: g^{-1}(\sigma) \rightarrow \sigma$. Surely $G_{\sigma}^{\prime}$ is a $K$-modification of $g$ on $g^{-1}(\sigma)$ and $G_{\sigma}^{\prime}$ equals $F_{n}$ on $g^{-1}(\sigma) \cap Y_{n}=g^{-1}(\partial \sigma)$. Furthermore, $G_{\sigma}^{\prime}$ equals $f$ on $g^{-1}(\sigma) \cap X$.

We define the function $F_{n+1}: Y_{n+1} \rightarrow|K|_{C W}$ to be $F_{n} \cup \bigcup\left\{G_{\sigma}^{\prime} \mid \sigma \in\right.$ $\left.K^{(n+1)} \backslash K^{(n)}\right\}$. The conditions $F_{n+1}\left|Y_{n+1} \cap X=f\right| Y_{n+1} \cap X: Y_{n+1} \cap X \rightarrow$ $|L|_{C W}, F_{n+1}$ is a $K$-modification of $g \mid Y_{n+1}$, and $F_{n+1} \mid Y_{n}=F_{n}: Y_{n} \rightarrow$ $|K|_{C W}$ are manifest from the construction. We need to check the continuity.

Let $y \in Y_{n+1}$. Then $g\left(U_{y}\right) \subset\left|K_{y}\right|_{C W}$, where $K_{y}$ is a finite subcomplex of $K$. So $F_{n+1}\left(U_{y}\right) \subset\left|K_{y}\right|_{C W}$ and $F_{n+1}$ is continuous on $g^{-1}(\sigma) \cap U_{y}$ for each $\sigma \in K_{y}$. So $F_{n+1}$ is continuous on $U_{y}$.

Finally, we set $F=\bigcup_{n=0}^{\infty} F_{n}: \bigcup_{n=0}^{\infty} Y_{n}=Y \rightarrow|K|_{C W}$. This well-defined function is continuous because for each $y$, there exists $n$ such that $F=F_{n}$ on $U_{y}$. Surely $F$ is a $K$-modification of $g$ and $\left.F|X=f: X \rightarrow| L\right|_{C W}$.

4.7. Lemma. Let $X$ be a subspace of a normal space $Y, L$ be a subcomplex of a locally finite-dimensional simplicial complex $K$, and $f:(Y, X) \rightarrow$ $\left(|K|_{C W},|L|_{C W}\right)$ be a map such that $\left.f|X: X \rightarrow| L\right|_{C W}$ is L-irreducible. Assume that for each $y \in Y$, there exists a finite subcomplex $K_{y}$ of $K$ and a neighborhood $U_{y}$ of $Y$ such that $f\left(U_{y}\right) \subset\left|K_{y}\right|_{C W}$. Then there exists a subcomplex $M$ of $K$ with $L \subset M$ and a $K$-modification $g: Y \rightarrow|M|_{C W} \subset$ $|K|_{C W}$ of $f$ such that $g|X=f| X: X \rightarrow|L|_{C W}$, and $g$ is $M$-irreducible.

The proof of this lemma essentially mirrors that of Lemma 7 of [M-U], so we shall not provide one. The main difference is that here the entire complex $K$ is locally finite-dimensional, whereas in [M-U], the complex was locally finite-dimensional perhaps only outside $L$.

4.8. Lemma. Let $N$ be a simplicial complex, $\mathcal{T}$ be a topology for $|N|$, and $f: X \rightarrow|N|_{\mathcal{T}}$ be a map. If $g: X \rightarrow|N|_{\mathcal{T}}$ is an $N$-modification of $f$, then $g^{-1} \operatorname{st}(U, N) \subset f^{-1} \operatorname{st}(U, N)$ for all $U \in N^{(0)}$, and hence $g^{-1}(\operatorname{st} N)$ refines $f^{-1}($ st $N)$.

Pro of. Suppose $x \in g^{-1} \operatorname{st}(U, N)$; then $g(x)$ lies in the interior of a simplex $\tau$ of $N$, where $U$ is a vertex of $\tau$. Now assume $f(x) \notin \operatorname{st}(U, N)$. Let $\sigma$ denote the simplex of $N$ such that $f(x) \in \operatorname{int} \sigma$; then $U$ is not a vertex of $\sigma$, but $g(x) \in \sigma$. This yields a contradiction since $\tau$ must be a face of $\sigma$.

4.9. Definition. Let $\mathcal{W}$ be an open cover of a closed subspace $X$ of a space $Y$. An expansion of $\mathcal{W}$ in $Y$ is a function $\theta: \mathcal{W} \rightarrow \mathcal{U}$, where $\mathcal{U}$ is a collection of open subsets of $Y$ such that

(1) $\theta(W) \cap X=W$ for all $W \in \mathcal{W}$. 
Let us say that the expansion is progressive if

(2) $U \cap X=\emptyset$ whenever $U \in \mathcal{U} \backslash \theta(\mathcal{W})$.

Whenever we have an expansion $\theta: \mathcal{W} \rightarrow \mathcal{U}$, we shall identify $N(\mathcal{W})$ as a subcomplex of $N(\mathcal{U})$ using the vertex map $\theta$ to induce the simplicial injection.

Using II 18.3 and IV 4.2 of [Hu] one can prove the following.

4.10. Lemma. Let $\mathcal{W}$ be a locally finite open cover of a closed subspace $X$ of a metric space $Y$. Let $\mathcal{R}$ be an open cover of $Y$ and suppose $\lambda: \mathcal{W} \rightarrow \mathcal{R}$ is a function with the property that $W \subset \lambda(W)$ for each $W \in \mathcal{W}$. Then there exists a progressive expansion $\theta: \mathcal{W} \rightarrow \mathcal{U}$ of $\mathcal{W}$ into $Y$ such that $\mathcal{U}$ is a locally finite collection in $Y$ and $W \subset \theta(W) \subset \lambda(W)$ for each $W \in \mathcal{W}$. Indeed, we may additionally choose $\mathcal{U}$ so that $\mathcal{U}$ refines $\mathcal{R}$ and $\mathcal{U}$ is a cover of $Y$.

4.11. Definition. Let $X$ be a metric space, and $\mathcal{U}$ be a locally finite open cover of $X$. Then the standard $\mathcal{U}$-barycentric map $b_{\mathcal{U}}: X \rightarrow|N(\mathcal{U})|_{C W}$ is defined as follows. For each $U \in \mathcal{U}$, let $g_{U}: X \rightarrow \mathbb{R}$ be given by $g_{U}(x)=$ $d(x, X \backslash U)$. We take the barycentric coordinate $\beta_{U}$ of $b_{\mathcal{U}}(x)$ to be

$$
\beta_{U}=g_{U}(x) / \sum\left\{g_{W}(x) \mid W \in \mathcal{U}\right\} .
$$

It is easy to check that $b_{\mathcal{U}}$ is indeed a $\mathcal{U}$-barycentric map.

4.12. Lemma. Let $X$ be a closed subspace of a metric space $Y, \mathcal{W}$ be a locally finite open cover of $X, \mathcal{U}$ be a locally finite open cover of $Y$, and $\theta: \mathcal{W} \rightarrow \mathcal{U}$ be a progressive expansion of $\mathcal{W}$ in $Y$. Using Definition 4.9, treat $N(\mathcal{W})$ as a subcomplex of $N(\mathcal{U})$. Then $\left.b_{\mathcal{U}}\left|X=b_{\mathcal{W}}: X \rightarrow\right| N(\mathcal{W})\right|_{C W} \subset$ $|N(\mathcal{U})|_{C W}$.

4.13. Lemma. Let $\mathcal{U}$ be a locally finite open cover of a metric space $X$. Then there exists an open cover $\mathcal{V}=\left\{V_{U} \mid U \in \mathcal{U}\right\}$ such that $V_{U} \subset U$ for each $U \in \mathcal{U}$ (a so-called "precise" refinement) and so that there exists a $\mathcal{V}$-normal map $f: X \rightarrow|N(\mathcal{V})|_{C W}$.

Proof. The reader may obtain a justification of this using 4.11, 4.3, and 4.5 .

5. Dowker systems, fine normal extensions. We want to state a result which is implicit in the proof of Lemma 3.2 of [Do]. One may consult the proof of Lemma 5 of $[\mathrm{M}-\mathrm{U}]$ to see another description of Dowker's construction. We leave it to the reader to verify details.

5.1. Lemma. There exists a triple $\left(\Phi, \varphi, \varphi_{0}\right)$, where to every simplicial complex $L, \Phi$ assigns an open cover $\Phi(L)$ of $|L|_{C W}$ and $\varphi$ assigns a function $\varphi(L): \Phi(L) \rightarrow L^{(0)}$ such that 
(1) $\Phi(L)$ is locally finite and has locally finite-dimensional nerve, and

(2) if $U \in \Phi(L)$, then $U \subset \operatorname{st}(\varphi(L)(U), L)$.

The element $\varphi_{0}$ has the property that if $L$ is a subcomplex of a simplicial complex $K$, then $\varphi_{0}=\varphi_{0}(L, K)$ is a function $\varphi_{0}: \Phi(L) \rightarrow \Phi(K)$ which is a progressive expansion of $\Phi(L)$ in $|K|_{C W}$ having the property that $\varphi(L)(U)=$ $\varphi(K)\left(\varphi_{0}(U)\right) \in L^{(0)}$ whenever $U \in \Phi(L)$.

5.2. Definition. We call a triple $\left(\Phi, \varphi, \varphi_{0}\right)$ as in Lemma 5.1 a Dowker system.

We fix a Dowker system $\left(\Phi, \varphi, \varphi_{0}\right)$ for the rest of this paper.

5.3. Definition. Let $X$ be a metric space and $\mathcal{R}$ be a locally finite open cover of $X$. Let us use $\Phi(\mathcal{R})$ to denote $\Phi(N(\mathcal{R})$ ). For each $A \in \Phi(\mathcal{R})$, let $T_{A}=b_{\mathcal{R}}^{-1}(A)$. Then the refinement $\psi(\mathcal{R})$ of $\mathcal{R}$ is $\left\{T_{A} \mid A \in \Phi(\mathcal{R})\right\}$, an indexed open cover of $X$. We shall denote its nerve as $\Sigma(\mathcal{R})$. Since $\Phi(\mathcal{R})$ has locally finite-dimensional nerve, it is not difficult to see that $\Sigma(\mathcal{R})$ is locally finite-dimensional. Similarly, $\psi(\mathcal{R})$ is an indexed locally finite open cover of $X$ which refines $\mathcal{R}$.

Using (2) of Lemma 5.1 and the fact that $b_{\mathcal{R}}$ is $\mathcal{R}$-barycentric, one deduces that $\psi(\mathcal{R})$ is a refinement of $\mathcal{R}$ in a special way.

5.4. Lemma. For each $T_{A} \in \psi(\mathcal{R})$, that is, for each $A \in \Phi(\mathcal{R})$, one has $T_{A} \subset b_{\mathcal{R}}^{-1} \operatorname{st}(\varphi(N(\mathcal{R}))(A), N(\mathcal{R})) \subset \varphi(N(\mathcal{R}))(A) \in N(\mathcal{R})^{(0)}=\mathcal{R}$.

Proof. This is true because $T_{A}=b_{\mathcal{R}}^{-1}(A)$, and hence by (2) of Lemma $5.1, A \subset \operatorname{st}(\varphi(N(\mathcal{R}))(A), N(\mathcal{R}))$.

By the absolute version of Theorem 4.3, there exists a subcomplex $E(\mathcal{R})$ of $\Sigma(\mathcal{R})$ and a map $h_{0}: X \rightarrow|E(\mathcal{R})|_{C W} \subset|\Sigma(\mathcal{R})|_{C W}$ which is $E(\mathcal{R})$-irreducible and is a $\Sigma(\mathcal{R})$-modification of $b_{\psi(\mathcal{R})}$. By Lemma 4.8, $h_{0}^{-1} \operatorname{st}(V, \Sigma(\mathcal{R}))=h_{0}^{-1} \operatorname{st}(V, E(\mathcal{R})) \subset b_{\psi(\mathcal{R})}^{-1} \operatorname{st}(V, \Sigma(\mathcal{R})) \subset V$ for each $V \in$ $E(\mathcal{R})^{(0)} \subset \Sigma(\mathcal{R})^{(0)}=\psi(\mathcal{R})$.

5.5. Definition. Let us refer to $E(\mathcal{R}) \subset \Sigma(\mathcal{R})$ as a principal complex of $\mathcal{R}$ and to any $E(\mathcal{R})$-irreducible map $h_{0}: X \rightarrow|E(\mathcal{R})|_{C W}$ which is a $\Sigma(\mathcal{R})$ modification of the standard $\psi(\mathcal{R})$-barycentric map $b_{\psi(\mathcal{R})}: X \rightarrow|\Sigma(\mathcal{R})|_{C W}$ as a principal map.

5.6. Lemma. Let $\mathcal{R}$ be a locally finite open cover of a metric space $X$. Then there is always a principal complex $E(\mathcal{R}) \subset \Sigma(\mathcal{R})$ of $\mathcal{R}$ and a principal map $h_{0}: X \rightarrow|E(\mathcal{R})|_{C W}$. For such $h_{0}$, one observes that $h_{0}^{-1}$ st $E(\mathcal{R})$ refines $\psi(\mathcal{R})$ and hence $\mathcal{R}$. Indeed, $h_{0}^{-1} \operatorname{st}(V, E(\mathcal{R})) \subset V$ for each $V \in E(\mathcal{R})^{(0)} \subset \Sigma(\mathcal{R})^{(0)}=\psi(\mathcal{R})$. Furthermore, each principal complex is locally finite-dimensional. 
5.7. Definition. We shall call each open cover $\mathcal{W}=h_{0}^{-1}$ st $E(\mathcal{R})$ as in Lemma 5.6 a principal refinement of $\mathcal{R}$ associated with $E(\mathcal{R})$. We usually treat $E(\mathcal{R})$ as the nerve of $\mathcal{W}$ as in Lemma 4.5, so the nerve of $\mathcal{W}$ is locally finite-dimensional.

5.8. Definition. There is an induced projection $\lambda_{h_{0}}: \mathcal{W} \rightarrow \mathcal{R}$ given as follows. Let $W=h_{0}^{-1} \operatorname{st}(V, E(\mathcal{R}))$; then $V$ is uniquely determined and $W \subset V \in \psi(\mathcal{R})$. Now such $V=T_{A}$ for some unique $A \in \Phi(\mathcal{R})=\Phi(N(\mathcal{R}))$. By Lemma 5.4, $T_{A} \subset \varphi(N(\mathcal{R}))(A) \in \mathcal{R}$. We define $\lambda_{h_{0}}(W)=\varphi(N(\mathcal{R}))(A)$. Note that $W \subset \lambda_{h_{0}}(W)$, so $\lambda_{h_{0}}$ is a projection.

We are now going to develop some concepts leading up to our next lemma. Let $\mathcal{R}$ be a locally finite open cover of a closed subspace $X$ of a metric space $Y$, let $\mathcal{S}$ be a locally finite open cover of $Y$, and let $\mu: \mathcal{R} \rightarrow \mathcal{S}$ be a progressive expansion of $\mathcal{R}$ in $Y$. According to Definition 4.9, $\mu$ induces a simplicial injection of $N(\mathcal{R})$ into $N(\mathcal{S})$, by which we take $N(\mathcal{R})$ to be a subcomplex of $N(\mathcal{S})$.

Using Lemma 5.1, we have the respective open covers $\Phi(\mathcal{R})$ and $\Phi(\mathcal{S})$ of $|N(\mathcal{R})|_{C W}$ and $|N(\mathcal{S})|_{C W}$ and the progressive expansion $\varphi_{0}=$ $\varphi_{0}(N(\mathcal{R}), N(\mathcal{S})): \Phi(\mathcal{R}) \rightarrow \Phi(\mathcal{S})$ in $|N(\mathcal{S})|_{C W}$. Also, there are functions $\varphi(\mathcal{R})=\varphi(N(\mathcal{R})): \Phi(\mathcal{R}) \rightarrow N(\mathcal{R})^{(0)}$ and $\Phi(\mathcal{S})=\varphi(N(\mathcal{S})): \Phi(\mathcal{S}) \rightarrow$ $N(\mathcal{S})^{(0)}$ such that $\varphi(\mathcal{R})(U)=\varphi(\mathcal{S})\left(\varphi_{0}(U)\right) \in L^{(0)}$ whenever $U \in \Phi(\mathcal{R})$. Additionally, (1), (2) of Lemma 5.1 are satisfied.

By Lemma 4.12, $\left.b_{\mathcal{S}}|X: X \rightarrow| N(\mathcal{R})\right|_{C W} \subset|N(\mathcal{S})|_{C W}$ is the standard $\mathcal{R}$-barycentric map $b_{\mathcal{R}}$. Applying Definition 5.3 we get the locally finite (indexed) open covers $\psi(\mathcal{R})$ and $\psi(\mathcal{S})$ of $X$ and $Y$ respectively, giving rise to the nerves $\Sigma(\mathcal{R}), \Sigma(\mathcal{S})$, which are locally finite-dimensional.

Assume that $\psi(\mathcal{R})=\left\{T_{A} \mid A \in \Phi(\mathcal{R})\right\}$ and $\psi(\mathcal{S})=\left\{S_{B} \mid B \in \Phi(\mathcal{S})\right\}$. This means that $\left(b_{\mathcal{S}} \mid X\right)^{-1}(A)=T_{A}$ and $b_{\mathcal{S}}^{-1}(B)=S_{B}, A \in \Phi(\mathcal{R}), B \in \Phi(\mathcal{S})$. Since $\varphi_{0}$ is a progressive expansion, $A=\varphi_{0}(A) \cap|N(\mathcal{R})|$, so $T_{A}=S_{\varphi_{0}(A)} \cap X$. If $B \in \Phi(\mathcal{S}) \backslash \varphi_{0}(\Phi(\mathcal{R}))$, then since $\varphi_{0}$ is progressive, we have $B \cap|N(\mathcal{R})|=\emptyset$. Hence $S_{B} \cap X=\emptyset$. This yields an (indexed) progressive expansion $\varphi_{1}$ : $\psi(\mathcal{R}) \rightarrow \psi(\mathcal{S})$ in $Y$ given by $\varphi_{1}\left(T_{A}\right)=S_{\varphi_{0}(A)}$. By means of this expansion $\varphi_{1}$, let us treat $\Sigma(\mathcal{R})$ as a subcomplex of $\Sigma(\mathcal{S})$ (use an indexed version of Definition 4.9).

By Lemma 4.12, $\left.b_{\psi(\mathcal{S})}\left|X=b_{\psi(\mathcal{R})}: X \rightarrow\right| \Sigma(\mathcal{R})\right|_{C W} \subset|\Sigma(\mathcal{S})|_{C W}$. Applying Lemma 5.6, let $E(\mathcal{R})$ be a principal complex of $\mathcal{R}$ and $h_{0}: X \rightarrow$ $|E(\mathcal{R})|_{C W}$ be a principal map. According to Lemma 4.6, there exists a map $F: Y \rightarrow|\Sigma(\mathcal{S})|_{C W}$ which is a $\Sigma(\mathcal{S})$-modification of $b_{\psi(\mathcal{S})}$ and such that $\left.F\left|X=h_{0}: X \rightarrow\right| \Sigma(\mathcal{R})\right|_{C W}$. Next apply Lemma 4.7 to $F$ to get a subcomplex $E(\mathcal{S})$ of $\Sigma(\mathcal{S})$ such that $E(\mathcal{R}) \subset E(\mathcal{S})$, and a $\Sigma(\mathcal{S})$-modification $h: Y \rightarrow|E(\mathcal{W})|_{C W} \subset|\Sigma(\mathcal{S})|_{C W}$ of $F$ such that $h|X=F| X=h_{0}: X \rightarrow$ 
$|E(\mathcal{R})|_{C W}$ and $h$ is $E(\mathcal{S})$-irreducible. Note that $E(\mathcal{S})$ is a principal complex of $\mathcal{S}$ with $h$ an associated principal map.

Let $\mathcal{W}=h_{0}^{-1} \operatorname{st} E(\mathcal{R})$ and $\mathcal{U}=h^{-1} \operatorname{st} E(\mathcal{S})$ be the respective principal refinements of $\mathcal{R}$ and $\mathcal{S}$. We are going to obtain a certain progressive expan$\operatorname{sion} \theta: \mathcal{W} \rightarrow \mathcal{U}$ in $Y$ which will have the property that for each $W \in \mathcal{W}$ $\mu \circ \lambda_{h_{0}}(W)=\lambda_{h} \circ \theta(W) \in \mathcal{S}$.

Let $W \in \mathcal{W}$ be arbitrary. Then $W=h_{0}^{-1} \operatorname{st}(V, E(\mathcal{R}))$ for some $V \in$ $E(\mathcal{R})^{(0)} \subset E(\mathcal{S})^{(0)} \subset \Sigma(\mathcal{S})^{0}=\psi(\mathcal{S})$. We define $\theta(W)=h^{-1} \operatorname{st}(V, E(\mathcal{S}))$ $\subset b_{\psi(\mathcal{S})}^{-1} \operatorname{st}(V, E(\mathcal{S}))$. Of course, $\theta(W) \in \mathcal{U}$. Since $\operatorname{st}(V, E(\mathcal{S})) \cap|E(\mathcal{R})|=$ $\operatorname{st}(V, E(\mathcal{R}))$ and $h(X) \subset|E(\mathcal{R})|$, it follows that $\theta(W) \cap X=h^{-1} \operatorname{st}(V, E(\mathcal{S})) \cap$ $X=W$. For any $V \in E(\mathcal{S})^{(0)} \backslash E(\mathcal{R})^{(0)}$, st $(V, E(\mathcal{S})) \cap|E(\mathcal{R})|=\emptyset$ and so $h^{-1} \operatorname{st}(V, E(\mathcal{S})) \cap X=\emptyset$. This shows that for any $B \in \mathcal{U} \backslash \theta(\mathcal{W}), B \cap X=\emptyset$. We therefore conclude that $\theta: \mathcal{W} \rightarrow \mathcal{U}$ is a progressive expansion of $\mathcal{W}$ in $Y$.

By Definition $5.8, \lambda_{h_{0}}(W)=\varphi(N(\mathcal{R}))(A) \in \mathcal{R}$ for a certain unique $A \in \Phi(\mathcal{R})$, where $V=T_{A}$. Similarly $\lambda_{h} \circ \theta(W)=\varphi(N(\mathcal{S}))(B) \in \mathcal{S}$ for a certain unique $B \in \Phi(\mathcal{S})$, where $V=S_{B}$. The identification of $\Sigma(\mathcal{R})$ as a subcomplex of $\Sigma(\mathcal{S})$ is obtained via the indexed progressive expansion $\varphi_{1}$, by which we see that $B=\varphi_{0}(A)$.

By Lemma 5.1, we have $\varphi(N(\mathcal{R}))(A)=\varphi(N(\mathcal{S}))\left(\varphi_{0}(A)\right) \in N(\mathcal{R})^{(0)} \subset$ $N(\mathcal{S})^{(0)}$. But $N(\mathcal{R})^{(0)}=\mathcal{R}$ and $N(\mathcal{S})^{(0)}=\mathcal{S}$, and the latter "inclusion" is in reality induced by the progressive expansion $\mu: \mathcal{R} \rightarrow \mathcal{S}$. Putting it formally, $\mu \circ \varphi(N(\mathcal{R}))(A)=\varphi(N(\mathcal{S}))\left(\varphi_{0}(A)\right)$, i.e., $\mu \circ \lambda_{h_{0}}(W)=\lambda_{h} \circ \theta(W)$, as required. Since $\theta(W) \subset \lambda_{h} \circ \theta(W)$, one has $\theta(W) \subset \mu \circ \lambda_{h_{0}}(W)$.

Let us put all this information into a lemma.

5.9. Lemma. Let $X$ be a closed subspace of a metric space $Y, \mathcal{R}$ be a locally finite open cover of $X, \mathcal{S}$ be a locally finite open cover of $Y$, and $\mu: \mathcal{R} \rightarrow \mathcal{S}$ be a progressive expansion of $\mathcal{R}$ in $Y$. Treat $\Sigma(\mathcal{R})$ as a subcomplex of $\Sigma(\mathcal{S})$ according to the preceding discussion using the vertex map $\varphi_{1}: \psi(\mathcal{R}) \rightarrow \psi(\mathcal{S})$. Then for each principal complex $E(\mathcal{R}) \subset \Sigma(\mathcal{R})$ of $\mathcal{R}$ and each principal map $h_{0}: X \rightarrow|E(\mathcal{R})|_{C W}$, there exists a principal complex $E(\mathcal{S}) \subset \Sigma(\mathcal{S})$ of $\mathcal{S}$ with $E(\mathcal{R}) \subset E(\mathcal{S})$ and there is a principal map $h: Y \rightarrow|E(\mathcal{S})|_{C W}$ such that $\left.h\left|X=h_{0}: X \rightarrow\right| E(\mathcal{R})\right|_{C W}$. Let $\mathcal{W}=$ $h_{0}^{-1}$ st $E(\mathcal{R})$ and $\mathcal{U}=h^{-1}$ st $E(\mathcal{S})$ be the respective principal refinements of $\mathcal{R}$ and $\mathcal{S}$. Then there exists a progressive expansion $\theta: \mathcal{W} \rightarrow \mathcal{U}$ such that for each $W \in \mathcal{W}, \mu \circ \lambda_{h_{0}}(W)=\lambda_{h} \circ \theta(W)$ and $\theta(W) \subset \mu \circ \lambda_{h_{0}}(W)$. (We note that this remains true for any choice of $E(\mathcal{S})$ and $h$.

5.10. Definition. A collection $\mathcal{C}$ of strongly normal covers of a space $X$ will be called central for $X$ if for each locally finite open cover $\mathcal{R}$ of $X$ consisting of non-empty sets, there exists a non-empty subset $\lambda_{\mathcal{C}}(\mathcal{R})$ of $\mathcal{C}$ such that for each $\mathcal{W} \in \lambda_{\mathcal{C}}(\mathcal{R}), \mathcal{W}$ refines $\mathcal{R}$. A projection $\lambda^{*}: \mathcal{W} \rightarrow \mathcal{R}$, in this case, will be called a central projection. If for each $\mathcal{W}$-normal map 
$f: X \rightarrow|N(\mathcal{W})|_{C W}$ there is prescribed a central projection $\lambda_{f}^{*}: \mathcal{W} \rightarrow \mathcal{R}$ then we shall say that $\mathcal{C}$ is projectedly central. We shall abbreviate the latter to proj-central.

Now suppose $X$ is a closed subspace of a metric space $Y, \mathcal{C}$ is proj-central for $X$ and $\mathcal{D}$ is proj-central for $Y$. Assume that whenever $\mathcal{R}$ is a locally finite open cover of $X, \mathcal{S}$ is a locally finite open cover of $Y$ and $\mu: \mathcal{R} \rightarrow \mathcal{S}$ is a progressive expansion of $\mathcal{R}$ in $Y$, then for any $\mathcal{W} \in \lambda_{\mathcal{C}}(\mathcal{R})$ and any $\mathcal{W}$-normal map $f: X \rightarrow|N(\mathcal{W})|_{C W}$ there exists $\mathcal{U} \in \lambda_{\mathcal{D}}(\mathcal{S})$, a progressive expansion $\theta: \mathcal{W} \rightarrow \mathcal{U}$ in $Y$ and a $\mathcal{U}$-normal map $g: Y \rightarrow|N(\mathcal{U})|_{C W}$ with $\left.g|X=f: X \rightarrow| N(\mathcal{W})\right|_{C W} \subset|N(\mathcal{U})|_{C W}$ and for each $W \in \mathcal{W}, \theta(W) \subset$ $\mu \circ \lambda_{f}^{*}(W)=\lambda_{g}^{*} \circ \theta(W)$. Then we call $\mathcal{D}$ a fine normal extension of $\mathcal{C}$ in $Y$.

An application of Lemmas 5.6 and 5.9 yields the next theorem.

5.11. Theorem. Let $X$ be a closed subspace of a metric space $Y, \mathcal{C}$ be the collection of all principal refinements in $X$ and $\mathcal{D}$ be the collection of all principal refinements in $Y$. Then $\mathcal{D}$ is a fine normal extension of $\mathcal{C}$ in $Y$. (Here we take $\lambda_{\mathcal{C}}(\mathcal{R})$ to be the collection of all principal refinements of $\mathcal{R}$ and $\lambda_{f}^{*}$ to be the induced projection of 5.8 , and similarly for $\mathcal{D}$.)

5.12. Note. Recall that a collection $\mathcal{D}$ of open covers of a space $Y$ is called cofinal if for each open cover $\mathcal{V}$ of $Y$, there exists $\mathcal{U} \in \mathcal{D}$ such that $\mathcal{U}$ refines $\mathcal{V}$. Thus in the absolute case of Definition 5.10, i.e., when $X=\emptyset$, the defining property of $\mathcal{D}$ is that $\mathcal{D}$ be central. In fact, though, it is sufficient that $\mathcal{D}$ be cofinal. Hence we have the following result.

5.13. Corollary. Let $Y$ be a metric space, $X=\mathcal{C}=\emptyset$, and $\mathcal{D}$ be a cofinal collection of strongly normal covers of $Y$. Then $\mathcal{D}$ is a fine normal extension of $\mathcal{C}$ in $Y$.

In conjunction with this, let us state the following fact which comes from an application of Lemma 5.6 and Definition 5.7.

5.14. Lemma. Let $Y$ be a metric space. Then the collection $\mathcal{D}$ of all principal refinements in $Y$ is cofinal.

6. Irreducible representations, definitive systems. We shall give the definition of a representation and its associated properties in the manner that will be required in this paper (ref. $[\mathrm{Ru}]$ ).

6.1. Definition. Let $\mathbf{P}=\left(P_{i}, \pi_{i, i+1}, \mathbb{N}\right)$ be an inverse sequence of spaces $P_{i}$ and $X$ be a space. A sequence $\left(g_{i}\right)_{i=1}^{\infty}$ of maps $g_{i}: X \rightarrow P_{i}$ with $\pi_{i, i+1}$ ○ $g_{i+1}=g_{i}$ for each $i \in \mathbb{N}$ will be called a representation of $X$ in $\mathbf{P}$ if the map $g=\prod_{i=1}^{\infty} g_{i}: X \rightarrow \prod_{i=1}^{\infty} P_{i}$ embeds $X$ onto a dense subspace of $\lim \mathbf{P}$. Call the representation polyhedral if each $P_{i}$, having a completely metrizable topology $\mathcal{T}_{i} \subset C W$ making it a locally finite-dimensional ANR, 
can be endowed with a locally finite-dimensional triangulation $K_{i}$ such that $\mathcal{T}_{i}$ induces $C W$ on each simplex of $K_{i}$ and st $K_{i}$ is an open cover of $\left|K_{i}\right|_{\mathcal{T}_{i}}$. If, in addition, each $g_{i}$ and bonding map $\pi_{i, i+1}$ is $K_{i}$-irreducible, let us say that the representation is irreducible, and simplicially irreducible if $\pi_{i, i+1}$ is simplicial from $K_{i+1}$ to some subdivision of $K_{i}$.

6.2. Theorem. Let $X$ be a metrizable space. Then $X$ has a simplicially irreducible representation $\left(g_{i}\right)_{i=1}^{\infty}$. If $X$ is completely metrizable, then we may choose $\left(g_{i}\right)_{i=1}^{\infty}$ and $\mathbf{P}$ so that $\left(g_{i}\right)_{i=1}^{\infty}$ is a simplicially irreducible representation of $X$ in $\mathbf{P}$ and $g=\prod_{i=1}^{\infty} g_{i}: X \rightarrow \lim \mathbf{P}$ is a homeomorphism. In case $X$ is compact, each $K_{i}$ is finite; if $X$ is separable, then each $K_{i}$ is countable and locally finite; in both instances the topology $\mathcal{T}_{i}$ is precisely $C W$.

Proof. Suppose $X$ is compact. Then each open cover $g_{i}^{-1} \operatorname{st}\left(K_{i}\right)$ has a finite subcover $\mathcal{V}_{i}$. We may treat $N\left(\mathcal{V}_{i}\right)$ as a subcomplex of $K_{i}$. There is a canonical map $h_{i}: X \rightarrow\left|N\left(\mathcal{V}_{i}\right)\right|_{C W}$ and since $\mathcal{T}_{i} \subset C W$, we may take $h_{i}$ as a map of $X$ to $\left|N\left(\mathcal{V}_{i}\right)\right|_{\mathcal{T}_{i}} \subset\left|K_{i}\right|_{\mathcal{T}_{i}}$. This latter map is a $K_{i}$-modification of the $K_{i}$-irreducible map $g_{i}: X \rightarrow\left|K_{i}\right|_{\mathcal{T}_{i}}$, which shows that $K_{i}$ must be finite.

In case $X$ is separable, first obtain a countable subcover $\mathcal{W}_{i}$ of $g_{i}^{-1} \operatorname{st}\left(K_{i}\right)$. Then find a precise locally finite refinement $\mathcal{V}_{i}$ of $\mathcal{W}_{i}$ whose nerve is locally finite, and proceed with the same argument as for the compact case.

In both instances, since $\mathcal{T}_{i} \subset C W$, the identity function from $\left|K_{i}\right|_{C W}$ to $\left|K_{i}\right|_{\mathcal{T}_{i}}$ is continuous. Now if $v$ is a vertex of $K_{i}$, then $\left|\overline{\operatorname{st}}\left(v, K_{i}\right)\right|$ inherits the same topology from $\mathcal{T}_{i}$ as it does from $C W$ since $\overline{\operatorname{st}}\left(v, K_{i}\right)$ is finite and both topologies agree on simplexes. Since st $K_{i}$ is an open cover of $\left|K_{i}\right|_{\mathcal{T}_{i}}$, the identity function from $\left|K_{i}\right|_{\mathcal{T}_{i}}$ to $\left|K_{i}\right|_{C W}$ is also continuous. Therefore $\mathcal{T}_{i}=C W$.

We delay the remainder of our proof of this theorem, as we need some preliminaries.

Let $K$ be a simplicial complex and $f: X \rightarrow|K|$ be a function. Surely if $\mathcal{T}$ is a topology for $|K|, f: X \rightarrow|K|_{\mathcal{T}}$ is continuous, and st $K$ is an open cover of $|K|_{\mathcal{T}}$, then $f^{-1}($ st $K)$ is an open cover of $X$. If $f: X \rightarrow|K|_{\mathcal{T}}$ happens to be also a $\mathcal{U}$-barycentric map, where $K$ is the nerve of $\mathcal{U}$, then $\mathcal{U}=f^{-1}$ (st $\left.K\right)$.

Suppose we have a collection of functions $\pi_{i, i+1}: X_{i+1} \rightarrow X_{i}, i \in \mathbb{N}$. Then we shall denote compositions of the form $\pi_{i, i+1} \circ \ldots \circ \pi_{k, k+1}$ as $\pi_{i, k+1}$.

6.3. Definition (compare with 3.2 of $[\mathrm{J}-\mathrm{R}]$ ). Suppose we are given a metric space $X$ and a sequence $P_{1}, P_{2}, \ldots$ of polyhedra with respective topologies $\mathcal{T}_{1}, \mathcal{T}_{2}, \ldots$ and locally finite-dimensional triangulations $K_{1,0}, K_{2,0}, \ldots$, such that for each $i \in \mathbb{N}$ :

(1) there is a sequential star-uniformity $\mathcal{B}_{i}$ for $P_{i}=\left|K_{i, 0}\right|$ determined by $\left\{K_{i, j}\right\}_{j=0}^{\infty}$ and yielding the topology $\mathcal{T}_{i}$ for $P_{i}$;

(2) there is a map $f_{i}: X \rightarrow P_{i}$; 
(3) the cover $f_{i+1}^{-1}$ (st $\left.K_{i+1,0}\right)$ refines $f_{i}^{-1}\left(\right.$ st $\left.K_{i, 1}\right)$;

(4) $\operatorname{mesh}\left(f_{i}^{-1}\left(\operatorname{st} K_{i, 0}\right)\right)<1 / 2^{i}$;

(5) there are maps $\tau_{i, i+1}, \pi_{i, i+1}: P_{i+1} \rightarrow P_{i}$ and $h_{i}: P_{i} \rightarrow P_{i}$ such that for some $\kappa(i) \in \mathbb{N}, h_{i}$ is a $K_{i, \kappa(i)}$-modification of the identity, $\tau_{i, i+1}$ is simplicial from $K_{i+1,0}$ to $K_{i, \kappa(i)}$ and is induced by a projection of $f_{i+1}^{-1}$ (st $K_{i+1,0}$ ) to $f_{i}^{-1}\left(\right.$ st $\left.K_{i, \kappa(i)}\right)$, and $\pi_{i, i+1}=h_{i} \circ \tau_{i, i+1}$;

(6) whenever $k \in \mathbb{N}$, there exist $l \in \mathbb{Z}_{>0}, m, p \in \mathbb{N}, m, p>i$, such that
(a) st $K_{i+1, l}$ refines $\pi_{i, i+1}^{-1}\left(\right.$ st $\left.K_{i, k}\right)$,
(b) st $K_{m, 0}$ refines $\tau_{i, m}^{-1}$ (st $K_{i, k}$ ), and
(c) $f_{p}^{-1}\left(\right.$ st $\left.K_{p, 0}\right)$ refines $f_{i}^{-1}\left(\right.$ st $\left.K_{i, k}\right)$.

Then we shall say that these data determine a definitive system $\mathcal{S}$ for $X$ or that $X$ supports the definitive system $\mathcal{S}$. Each such definitive system induces an inverse sequence $\mathbf{P}=\left(P_{i}, \pi_{i, i+1}, \mathbb{N}\right)$ of polyhedra $P_{i}$ with topologies $\mathcal{T}_{i}$. According to Proposition 3.5, these topologies are metrizable, the uniformities $\mathcal{B}_{i}$ are complete, and $P_{i}$ is in fact an ANR. We endow $\prod_{i=1}^{\infty} P_{i}$ with the (complete) product uniformity from the $\mathcal{B}_{i}$ 's and $\lim \mathbf{P}$ with the uniformity inherited from this product uniformity.

Let us state that in the current paper, the maps $h_{i}$ in (5) will always be the identity, and thus in the applications herein will not even be mentioned because in this situation $\pi_{i, i+1}$ will be the same as $\tau_{i, i+1}$. But in future applications of these techniques, this will not be the case, and we have included this information for that purpose.

6.4. Lemma. Given a definitive system $\mathcal{S}$ for $X$ and $i, k \in \mathbb{N}$, the following properties are satisfied:

(7) $\pi_{i, i+k}$ is a $K_{i, \kappa(i)}$-modification of $\tau_{i, i+k}$, and

(8) $\pi_{i, i+k} \circ f_{i+k}$ is a $K_{i, \kappa(i)}$-modification of $f_{i}$.

Proof. Suppose that (7) is true and, in addition,

(9) $\tau_{i, i+k} \circ f_{i+k}$ is a $K_{i, \kappa(i)}$-modification of $f_{i}$.

For any $x \in X, \tau_{i, i+k}\left(f_{i+k}(x)\right)$ lies in a face $\sigma_{0}$ of the simplex $\sigma$ of $K_{i, \kappa(i)}$ where $f_{i}(x) \in \operatorname{int} \sigma$. But then by $(7), \pi_{i, i+k}\left(f_{i+k}(x)\right)$ lies in $\sigma_{0}$, so (8) is true. We therefore intend to prove that (7) and (9) hold.

To prove (7), note first that it is certainly true in case $k=1$. We can say even more since $\tau_{i, i+1}$ is simplicial from $K_{i+1,0}$ to $K_{i, \kappa(i)}$ : if $\sigma$ is a simplex of $K_{i+1,0}$, then $\pi_{i, i+1}(\sigma)$ is contained in the simplex $\tau=\tau_{i, i+1}(\sigma)$ of $K_{i, \kappa(i)}$.

Let $k \in \mathbb{N}$ and assume inductively that for any simplex $\sigma$ of $K_{i+k, 0}$, $\pi_{i, i+k}(\sigma)$ is contained in the simplex $\tau=\tau(\sigma)$ of $K_{i, \kappa(i)}$ which is minimal with respect to containing $\tau_{i, i+k}(\sigma)$, i.e., which contains it and has the same dimension. Now let $\sigma_{0}$ be a simplex of $K_{i+k+1,0}$ and consider the simplex $\gamma=\tau_{i+k, i+k+1}\left(\sigma_{0}\right)$ of $K_{i+k, \kappa(i+k)}$. Then $\gamma$ is contained in a minimal simplex 
$\sigma$ of $K_{i+k, 0}$. Surely $\pi_{i+k, i+k+1}\left(\sigma_{0}\right) \subset \gamma \subset \sigma$. Hence by the inductive assumption, $\pi_{i, i+k}\left(\pi_{i+k, i+k+1}\left(\sigma_{0}\right)\right)$ is contained in $\tau(\sigma)$. However, $\tau(\sigma)$ is the same as $\tau\left(\sigma_{0}\right)$ by consideration of the fact that each $\tau_{j, j+1}$ is simplicial and that $\gamma$ contains at least one point of int $\sigma$. So our statement above is true.

We get (7) from this easily. Consider $x \in P_{i+k}$ and let $\sigma$ be the simplex of $K_{i+k, 0}$ with $x \in \operatorname{int} \sigma$. One sees that $\tau_{i, i+k}(x)$ lies in int $\tau(\sigma)$, for otherwise the simplex which is the image of $\tau_{i, i+k}(\sigma)$ would lie in a proper face of $\tau(\sigma)$. From the preceding, we get $\pi_{i, i+k}(x) \in \tau(\sigma)$, and we are finished with (7).

To prove (9), inductively assume that $\tau_{i, i+k} \circ f_{i+k}$ is a $K_{i, \kappa(i)}$-modification of $f_{i}$ and show that $\tau_{i, i+k+1} \circ f_{i+k+1}$ is also a $K_{i, \kappa(i)}$-modification of $f_{i}$. Let $x \in X$ and consider the simplex $\sigma_{0}$ of $K_{i+k, 0}$ such that $f_{i+k}(x)$ lies in $\operatorname{int} \sigma_{0}$. Let $\tau$ be the simplex of $K_{i, \kappa(i)}$ containing $f_{i}(x)$ in its interior. Then $\tau_{i, i+k}\left(f_{i+k}(x)\right)$ lies in the interior of some face $\delta$ of $\tau$. The simplicial structure of the bonding maps given by (5) yields that $\tau_{i, i+k}$ carries $\sigma_{0}$ into $\delta$. Hence our proof will be complete if we show that $\tau_{i+k, i+k+1}\left(f_{i+k+1}(x)\right)$ lies in $\sigma_{0}$.

Let $\gamma: f_{i+k+1}^{-1}\left(\right.$ st $\left.K_{i+k+1,0}\right) \rightarrow f_{i+k}^{-1}\left(\right.$ st $\left.K_{i+k, \kappa(i+k)}\right)$ be the projection of (5) which induces $\tau_{i+k, i+k+1}$. Denote $\gamma f_{i+k+1}^{-1}\left(\operatorname{st}\left(v, K_{i+k+1,0}\right)\right)$ as $f_{i+k}^{-1}(\operatorname{st}(\alpha(v)$, $\left.\left.K_{i+k, \kappa(i+k)}\right)\right)$, with $v$ a vertex of $K_{i+k+1,0}$; then $f_{i+k+1}^{-1}\left(\operatorname{st}\left(v, K_{i+k+1,0}\right)\right) \subset$ $f_{i+k}^{-1}\left(\operatorname{st}\left(\alpha(v), K_{i+k, \kappa(i+k)}\right)\right)$.

Let $x \in X$ and $\sigma$ be the simplex of $K_{i+k, \kappa(i+k)}$ such that $f_{i+k}(x)$ lies in $\operatorname{int}(\sigma)$, and let $w_{1}, \ldots, w_{r}$ be the vertices of $\sigma$. Now $\sigma \subset \sigma_{0}$ and, moreover, $w_{1}, \ldots, w_{r}$ are the only vertices $w$ of $K_{i+k, \kappa(i+k)}$ such that $x \in$ $f_{i+k}^{-1}\left(\operatorname{st}\left(w, K_{i+k, \kappa(i+k)}\right)\right)$.

The map $f_{i+k+1}$ carries $x$ into the interior of a simplex $\tau$ of $K_{i+k+1,0}$ with vertices, say, $v_{1}, \ldots, v_{r}$. Since $x \in f_{i+k+1}^{-1}\left(\operatorname{st}\left(v_{s}, K_{i+k+1,0}\right)\right), 1 \leq s \leq r$, it follows that $\alpha\left(v_{s}\right)$ must be an element of $w_{1}, \ldots, w_{k}$. Since $\tau_{i+k, i+k+1}$ is simplicial from $K_{i+k+1,0}$ to $K_{i+k, \kappa(i+k)}$, we get $\tau_{i+k, i+k+1}\left(f_{i+k+1}(x)\right) \in$ $\tau_{i+k, i+k+1}(\tau) \subset \sigma \subset \sigma_{0}$, as desired.

6.5. Lemma. Let $\mathbf{P}$ be the inverse sequence induced by a definitive system $\mathcal{S}$ for a metric space $X$ (with notation as in Definition 6.3). Then for each $i \in \mathbb{N},\left(\pi_{i, j} \circ f_{j}\right)_{j>i}$ is a Cauchy sequence of maps into the complete uniform space $\left(P_{i}, \mathcal{B}_{i}\right)$. Defining $g_{i}=\lim _{j \rightarrow \infty}\left(\pi_{i, j} \circ f_{j}\right): X \rightarrow P_{i}$ we find that $g_{i}$ is a $K_{i, \kappa(i)}$-modification of $f_{i}$ and $\pi_{i, i+1} \circ g_{i+1}=g_{i}$; furthermore, $g_{\mathcal{S}}=$ $\prod_{i=1}^{\infty} g_{i}: X \rightarrow \lim \mathbf{P}$ is an embedding of $X$ into $\lim \mathbf{P}$. If each $f_{i}: X \rightarrow P_{i}$ is $K_{i, 0}$-irreducible, then $\operatorname{dim} P_{i} \leq \operatorname{dim} X$ and $g_{\mathcal{S}}(X)$ is dense in $\lim \mathbf{P}$; if in addition the metric for $X$ is complete, then $g_{\mathcal{S}}(X)=\lim \mathbf{P}$.

Proof. Fix $i$ and $k$. Choose $m$ as in (6b), and suppose $j \geq m$. Let $x \in X$. We want to show that $\tau_{i, j}\left(f_{j}(x)\right), \tau_{i, m}\left(f_{m}(x)\right)$ lie in an element of st $K_{i, k}$. From this and 6.4(7) it follows that $\pi_{i, j}\left(f_{j}(x)\right), \pi_{i, m}\left(f_{m}(x)\right)$ lie in an element of $\mathrm{st}^{2} K_{i, k}$, which will prove that $\left(\pi_{i, j} \circ f_{j}\right)_{j>i}$ is a Cauchy sequence. 
Using (7), one sees that $\pi_{m, j}\left(f_{j}(x)\right)$ lies in a simplex $\sigma$ of $K_{m, \kappa(m)}$ having the property that $f_{m}(x)$ lies in $\operatorname{int}(\sigma)$. Hence there is a vertex $v$ of $\sigma$ such that $\pi_{m, j}\left(f_{j}(x)\right), f_{m}(x)$ lie in $\operatorname{st}\left(v, K_{m, \kappa(m)}\right)$. This implies that there is a vertex $w$ of $K_{m, 0}$ with $\pi_{m, j}\left(f_{j}(x)\right), f_{m}(x) \in \operatorname{st}\left(w, K_{m, 0}\right)$. Our choice of $m$ in (6b) yields that st $K_{m, 0}$ refines $\pi_{i, m}^{-1}\left(\right.$ st $\left.K_{i, k}\right)$. Thus, $\pi_{i, m}\left(\pi_{m, j}\left(f_{j}(x)\right)\right)=$ $\pi_{i, j}\left(f_{j}(x)\right)$ and $\pi_{i, m}\left(f_{m}(x)\right)$ lie in an element of st $K_{i, k}$, as needed.

From the definition of $g_{i}$ and (7), it is immediate that $g_{i}$ is a $K_{i, \kappa(i)^{-}}$ modification of $f_{i}$. Now $\pi_{i, i+1} \circ g_{i+1}=\pi_{i, i+1} \circ \lim _{l>i+1} \pi_{i+1, l} \circ f_{l}=$ $\lim _{l>i+1} \pi_{i, i+1} \circ \pi_{i+1, l} \circ f_{l}=\lim _{l>i} \pi_{i, l} \circ f_{l}=g_{i}$. This of course shows that the map $g_{\mathcal{S}}$ carries $X$ into $\lim \mathbf{P}$.

Suppose that $f_{i}$ is $K_{i, 0}$-irreducible. Since $g_{i}$ is a $K_{i, \kappa(i)}$-modification of $f_{i}$, it is also a $K_{i, 0}$-modification of $f_{i}$. Hence $g_{i}$ is surjective. So for any non-empty open subset $U$ of $P_{i}$, choose $x \in X$ with $g_{i}(x) \in U$. Then the thread $g_{\mathcal{S}}(x) \in \lim \mathbf{P}$ lies in the basic open set $\pi_{i}^{-1}(U)$ of $\lim \mathbf{P}$. Hence $g_{\mathcal{S}}(X)$ is dense in $\lim \mathbf{P}$.

Let us now prove that $g_{\mathcal{S}}$ is injective. Let $x, y \in X, x \neq y$. Using (4), choose $i$ so that $\operatorname{mesh}\left(f_{i}^{-1}\left(\right.\right.$ st $\left.\left.K_{i, 0}\right)\right)<d(x, y)$. Let $\sigma_{x}, \sigma_{y}$ be simplexes of $K_{i, 0}$ with $f_{i}(x) \in \operatorname{int} \sigma_{x}, f_{i}(y) \in \operatorname{int} \sigma_{y}$. We claim that $\sigma_{x} \cap \sigma_{y}=\emptyset$. If not, then there is a common vertex $v$ of $\sigma_{x}$ and $\sigma_{y}$ and $f_{i}(x), f_{i}(y) \in \operatorname{st}\left(v, K_{i, 0}\right)$. Then $x, y \in f_{i}^{-1}\left(\operatorname{st}\left(v, K_{i, 0}\right)\right)$, but since $\operatorname{diam}\left(f_{i}^{-1}\left(\operatorname{st}\left(v, K_{i, 0}\right)\right)\right)<d(x, y)$ this is impossible. Since $g_{i}$ is a $K_{i, 0}$-modification of $f_{i}$, we have $g_{i}(x) \in \sigma_{x}$, $g_{i}(y) \in \sigma_{y}$. Hence $g_{i}(x) \neq g_{i}(y)$, and $g_{\mathcal{S}}$ is injective.

To prove that $g_{\mathcal{S}}$ is an embedding and indeed a surjection if $g_{\mathcal{S}}(X)$ is dense in $\lim \mathbf{P}$ and the metric for $X$ is complete, we shall make use of uniformities. Let us designate by $\mathcal{U}_{i}$ the open cover $f_{i}^{-1}$ (st $\left.K_{i, 0}\right)$ of $X$.

Recall that st $K_{i, 1}$ star-refines st $K_{i, 0}$. From this and (3) we see that $\mathcal{U}_{i+1}$ star-refines $\mathcal{U}_{i}$. Taking into account $(4)$, we find that $\left\{\mathcal{U}_{i}\right\}$ is a base for a uniformity on $X$ yielding the metric topology. This uniformity is complete if the metric is complete. Now $\lim \mathbf{P}$ bears the uniformity inherited from the product uniformity, which is complete since $\lim \mathbf{P}$ is closed in $\prod_{i=1}^{\infty} P_{i}$, which is complete since each $\mathcal{B}_{i}$ is complete.

The maps $\pi_{i, i+1}$ are uniformly continuous because of (6a). The maps $f_{i}$ are uniformly continuous because of (6c). It follows then that each $g_{i}$, being the limit of a Cauchy sequence of uniformly continuous maps, is uniformly continuous. Since $\prod_{i=1}^{\infty} P_{i}$ has the product uniformity (uniformity of uniform convergence), we find that $g_{\mathcal{S}}$ is also uniformly continuous. If we can prove that $g_{\mathcal{S}}^{-1}: g_{\mathcal{S}}(X) \rightarrow X$ is uniformly continuous, then we shall know that $g_{\mathcal{S}}$ is a uniform embedding of $X$ onto a subset of $\lim \mathbf{P}$. Therefore in case $g_{\mathcal{S}}$ is dense in $\lim \mathbf{P}$ and the metric for $X$ is complete, we will have $g_{\mathcal{S}}(X)=\lim \mathbf{P}$, and our proof will be finished.

For an inverse sequence of uniform spaces, each with a given base for its uniformity, and such that the bonding maps are uniformly continuous, there 
is a base for the uniformity induced on the limit which comes from these data. In the current case this base consists of all sets $\pi_{i}^{-1}\left(\right.$ st $\left.K_{i, l}\right) \cap \lim P_{i}$, $l \in \mathbb{N}$, where $\pi_{i}: \lim \mathbf{P} \rightarrow P_{i}$ is the coordinate projection, and is connected to the fact that for each $i,\left\{\text { st } K_{i, l}\right\}_{l=1}^{\infty}$ is a base for the uniformity on $P_{i}$. The subspace $g_{\mathcal{S}}(X)$ of $\lim \mathbf{P}$ thus has a uniform base consisting of all sets $\pi_{i}^{-1}\left(\operatorname{st} K_{i, l}\right) \cap g_{\mathcal{S}}(X), i, l \in \mathbb{N}$.

To show that $g_{\mathcal{S}}^{-1}: g_{\mathcal{S}}(X) \rightarrow X$ is uniformly continuous, it is sufficient to prove that for each uniform base element $\mathcal{U}_{k}$ for $X$, there exist $i, l \in \mathbb{N}$ with the property that $W_{i, l}=\pi_{i}^{-1}\left(\right.$ st $\left.K_{i, l}\right) \cap g_{\mathcal{S}}(X)$ refines $\left(g_{\mathcal{S}}^{-1}\right)^{-1}\left(\mathcal{U}_{k}\right)=g_{\mathcal{S}}\left(\mathcal{U}_{k}\right)$. Since $g_{\mathcal{S}}$ is injective, it is sufficient to show that $g_{\mathcal{S}}^{-1}\left(W_{i, l}\right)$ refines $\mathcal{U}_{k}$. But $g_{\mathcal{S}}^{-1}\left(W_{i, l}\right)=g_{\mathcal{S}}^{-1}\left(\pi_{i}^{-1}\left(\right.\right.$ st $\left.\left.K_{i, l}\right)\right)$ and since $\pi_{i} g_{\mathcal{S}}=g_{i}$, this latter set is $g_{i}^{-1}\left(\right.$ st $\left.K_{i, l}\right)$. It remains then to prove that

(8) for each $k \in \mathbb{N}$ there exist $i, l \in \mathbb{N}$ with the property that $g_{i}^{-1}$ (st $K_{i, l}$ ) refines $\mathcal{U}_{k}=f_{k}^{-1}\left(\right.$ st $\left.K_{k, 0}\right)$.

Choose $i=k$ and $l=1$. Note that st $K_{k, \kappa(k)}$ star-refines st $K_{k, 0}$. Let $W \in$ st $K_{k, \kappa(k)}$ and consider $g_{k}^{-1}(W)$. For some $v \in K_{k, \kappa(k)}, W=\operatorname{st}\left(v, K_{k, \kappa(k)}\right)$ and st $\left(W\right.$, st $\left.K_{k, \kappa(k)}\right)$ lies in an element $V$ of st $K_{k, 0}$. We claim that $g_{k}^{-1}(W) \subset$ $f_{k}^{-1}(V)$.

To see this, suppose there exists $x \in g_{k}^{-1}(W) \backslash f_{k}^{-1}(V)$. Let $\tau$ be the simplex of $K_{k, \kappa(k)}$ with $g_{k}(x) \in \operatorname{int}(\tau)$ and $\sigma$ be the one with $f_{k}(x) \in \operatorname{int}(\sigma)$. Now $v$ is a vertex of $\tau$ and we know that $f_{k}(x) \notin \operatorname{st}\left(W\right.$, st $\left.K_{k, \kappa(k)}\right)$. Hence $\sigma \cap \tau=\emptyset$. But we have already shown that $g_{k}$ is a $K_{k, \kappa(k)}$-modification of $f_{k}$, so $g_{k}(x) \in \sigma$, a contradiction. This finalizes the proof.

6.6. Corollary. Let a metric space $X$ support a definitive system $\mathcal{S}$ where the maps $f_{i}$ in (2) are $K_{i, 0}$-irreducible. Then the sequence $\left(g_{i}\right)_{i=1}^{\infty}$ of maps $g_{i}: X \rightarrow \prod_{i=1}^{\infty} P_{i}$ is a simplicially irreducible representation of the space $X$ in the inverse sequence $\mathbf{P}=\left(P_{i}, \pi_{i, i+1}, \mathbb{N}\right)$ induced by the definitive system $\mathcal{S}$. Each $P_{i}=\left|K_{i, 0}\right|_{\mathcal{T}_{i}}$ is a completely metrizable ANR, $\mathcal{T}_{i} \subset C W$, $K_{i, 0}$ is locally finite-dimensional, st $K_{i}$ is an open cover of $\left|K_{i}\right|_{\mathcal{T}_{i}}, \operatorname{dim} P_{i} \leq$ $\operatorname{dim} X$, and $g_{\mathcal{S}}: X \rightarrow \lim \mathbf{P}$ is a homeomorphism if the metric for $X$ is complete. Consequently, Theorem 6.2 is true if every metric space supports such a definitive system.

Proof. Since each $K_{i, 0}$ is locally finite-dimensional and $\mathcal{T}_{i}$ is induced by a star-uniformity $\mathcal{B}_{i}$ on $P_{i}=\left|K_{i, 0}\right|$, Proposition 3.5 (vi) yields that $P_{i}$ is an ANR, (ii) gives us that $\mathcal{T}_{i} \subset C W$, and (i) that st $K_{i}$ is an open cover of $\left|K_{i}\right|_{\mathcal{T}_{i}}$. The preceding lemma shows that $\left(g_{i}\right)_{i=1}^{\infty}$ is a representation of $X$ in P. From Definition 6.3(5) one has the simplicial property. Now each $g_{i}$ is a $K_{i, \kappa(i)^{-}}$and hence $K_{i, 0}$-modification of $f_{i}$. Since $f_{i}$ is $K_{i, 0}$-irreducible, so is $g_{i}$. Since $\pi_{i, i+1} \circ g_{i+1}=g_{i}$, it is easily seen that if $\pi_{i, i+1}$ were not $K_{i, 0}$-irreducible then $g_{i}$ would not be either. Hence the corollary is true. 
As a result of this corollary, we will get Theorem 6.2 provided we can show that each metric space $X$ supports a definitive system $\mathcal{S}$ with the maps $f_{i}$ being $K_{i, 0}$-irreducible. This is going to be accomplished in the next section in Theorem 7.4.

7. Obtaining definitive systems. In this section we shall demonstrate that every metric space supports a definitive system. We shall do more though, by laying the groundwork for results to come in later sections. For a metric space $X$ and $\varepsilon>0$, let $\operatorname{Cov}(X, \varepsilon)$ be the open cover $X$ consisting of all open balls of radius $\varepsilon$.

7.1. Lemma. Let $X$ be a metric space and $\mathcal{W}_{1}, \mathcal{W}_{2}, \ldots$ be a sequence of strongly normal covers of $X$ having respective locally finite-dimensional nerves $L_{1,0}, L_{2,0}, \ldots$ Suppose that for each $i \in \mathbb{N}$,

(1) $\mathcal{W}_{i}$ refines $\operatorname{Cov}\left(X, 1 / 2^{i+2}\right)$,

(2) a $\mathcal{W}_{i}$-normal map $f_{i}: X \rightarrow\left|L_{i, 0}\right|_{C W}$ has been chosen,

(3) there is a collection $\left\{L_{i, j}\right\}_{j=0}^{\infty}$ of subdivisions of $L_{i, 0}$ which determines a sequential star-uniformity $\mathcal{B}_{i}$ for $P_{i}=\left|L_{i, 0}\right|$ with induced topology $\mathcal{T}_{i}$

(4) $f_{i+1}^{-1}$ (st $\left.L_{i+1,0}\right)$ refines $f_{i}^{-1}\left(\right.$ st $\left.L_{i, 1}\right)$,

(5) there is a simplicial map $\pi_{i, i+1}: L_{i+1,0} \rightarrow L_{i, 1}$ which is induced by a projection $\psi_{i, i+1}$ of $\mathcal{W}_{i+1}=f_{i+1}^{-1}$ (st $\left.L_{i+1,0}\right)$ to $f_{i}^{-1}$ (st $\left.L_{i, 1}\right)$,

(6) for all $j \in \mathbb{N}, \overline{\mathrm{st}} L_{i+1, j}$ refines $\pi_{i, i+1}^{-1}$ (st $L_{i, i+j}$ ), and

(7) $f_{i}^{-1}$ (st $\left.L_{i, 0}\right)$ refines $f_{s}^{-1}\left(\right.$ st $\left.L_{s, k}\right)$ whenever $s<i$ and $k \leq i+1$.

Then the data generated by these conditions satisfy the requirements for a definitive system $\mathcal{S}$ for $X$ as stated in Definition 6.3, where we replace $C W$ on $\left|L_{i, 0}\right|$ by $\mathcal{T}_{i}$ for each $i \in \mathbb{N}$ and where the maps $f_{i}$ are $L_{i, 0}$-irreducible $\left(h_{i}=\mathrm{id}\right.$ and $\left.\tau_{i, i+1}=\pi_{i, i+1}\right)$.

Pr o o f. Condition 6.3(1) is given by (3). Since by (2), $f_{i}: X \rightarrow\left|L_{i, 0}\right|_{C W}$ is $\mathcal{W}_{i}$-normal and by (ii), (iii) of Proposition 3.5, $\mathcal{T}_{i} \subset C W$ and $\mathcal{T}_{i}$ induces $C W$ on each simplex of $L_{i, 0}$, it follows that $f_{i}: X \rightarrow\left|L_{i, 0}\right|_{\mathcal{T}_{i}}=P_{i}$ is $\mathcal{W}_{i^{-}}$ normal. This shows that $f_{i}$ is $L_{i, 0}$-irreducible, so we have $6.3(2)$ with the additional condition requested above. One gets 6.3(3) from (4), and 6.3(4) from (1) and the fact that, $f_{i}$ being $\mathcal{W}_{i}$-normal, $f_{i}^{-1}$ (st $\left.L_{i, 0}\right)=\mathcal{W}_{i}$. Also, $6.3(5)$ comes directly from (5).

As for (6) of 6.3 , suppose $k \in \mathbb{N}$. To get (6a), suppose first that $k=1$. Then since $\pi_{i, i+1}: L_{i+1,0} \rightarrow L_{i, 1}$ is simplicial, it is easy to see that st $L_{i+1,0}$ refines $\pi_{i, i+1}^{-1}$ (st $L_{i, k}$ ), so choose $l=0$. If $k>1$, choose $l=k-1$. Then $i+l \geq k$, so surely $\operatorname{st}\left(L_{i, i+l}\right)$ refines st $L_{i, k}$, and hence $\pi_{i, i+1}^{-1}\left(\right.$ st $\left.L_{i, i+l}\right)$ refines $\pi_{i, i+1}^{-1}\left(\right.$ st $\left.L_{i, k}\right)$. However, (6) shows that st $L_{i+1, l}$ refines $\pi_{i, i+1}^{-1}\left(\operatorname{st} L_{i, i+l}\right)$. 
Now for $(6 \mathrm{~b})$ of 6.3 . We have just used the fact that st $L_{i+1,0}$ refines $\pi_{i, i+1}^{-1}\left(\right.$ st $\left.L_{i, 1}\right)$, so if $k=1$, then choose $m=i+1$. In case $k=2$ we see that st $L_{i+2,0}$ refines $\pi_{i+1, i+2}^{-1}\left(\right.$ st $\left.L_{i+1,1}\right)$. From (6) one deduces that st $L_{i+1,1}$ refines $\pi_{i, i+1}^{-1}\left(\right.$ st $\left.L_{i, i+1}\right)$, which surely refines $\pi_{i, i+1}^{-1}\left(\right.$ st $\left.L_{i, 2}\right)=\pi_{i, i+1}^{-1}\left(\right.$ st $\left.L_{i, k}\right)$. Hence st $L_{i+2,0}$ refines $\pi_{i+1, i+2}^{-1}\left(\pi_{i, i+1}^{-1}\left(\right.\right.$ st $\left.\left.L_{i, k}\right)\right)=\pi_{i, i+2}^{-1}\left(\operatorname{st} L_{i, k}\right)$. So in this case take $m=i+2$. In general, one chooses $m=i+k$; we leave the details to the reader.

Finally, we need to show (6c) of 6.3 . We must find $p \in \mathbb{N}, p>i$, such that $f_{p}^{-1}\left(\right.$ st $\left.L_{p, 0}\right)$ refines $f_{i}^{-1}\left(\right.$ st $\left.L_{i, k}\right)$. Take $p=i+k$. Then surely $i<p$ and $k \leq p+1$. So (7) applies and we get the needed relation.

7.2. Definition. Let $X$ be a metric space and $\mathcal{C}$ be a collection of strongly normal covers of $X$ each having locally finite-dimensional nerve and $\mathcal{F}=\left\{\left(\mathcal{W}_{i}, L_{i, j}, f_{i}, \mathcal{T}_{i}, \pi_{i, i+1}, \psi_{i, i+1}\right) \mid i \in \mathbb{N}, j \geq 0\right\}$ be a set of data satisfying the conditions of Lemma 7.1 , where $\mathcal{W}_{i} \in \mathcal{C}$ for each $i \in \mathbb{N}$. We shall call $\mathcal{F}$ a determining system for $\mathcal{C}$.

A sequence $\left\{\left(\mathcal{R}_{i}, \lambda_{i}, \psi_{i}\right) \mid i \in \mathbb{N}\right\}$ will be called a filtering for $\mathcal{F}$ if for each $i \in \mathbb{N}$,

(1) $\mathcal{R}_{i}$ is a locally finite open cover of $X$,

(2) $\mathcal{W}_{i}$ refines $\mathcal{R}_{i}$

(3) $\mathcal{R}_{i}$ refines $\operatorname{Cov}\left(X, 1 / 2^{i+2}\right)$,

(4) $\mathcal{R}_{i+1}$ refines $f_{s}^{-1}\left(\right.$ st $\left.L_{s, k}\right)$ whenever $s<i+1$ and $k \leq i+2$, and

(5) there are fixed projections $\lambda_{i}: \mathcal{W}_{i} \rightarrow \mathcal{R}_{i}$ and $\psi_{i}: \mathcal{R}_{i+1} \rightarrow f_{i}^{-1}\left(\right.$ st $\left.L_{i, 1}\right)$ such that $\psi_{i, i+1}$ of (5) of Lemma 7.1 is the composition $\psi_{i} \circ \lambda_{i+1}: \mathcal{W}_{i} \rightarrow$ $f_{i}^{-1}\left(\right.$ st $\left.L_{i, 1}\right)$.

7.3. Lemma. Let $X$ be a closed subspace of a metric space $Y$ and $\mathcal{C}, \mathcal{D}$ be respective collections of normal covers of $X$, each having locally finitedimensional nerve and such that $\mathcal{D}$ is a fine normal extension of $\mathcal{C}$ in $Y$. Let $\lambda_{\mathcal{C}}, \lambda_{\mathcal{L}}, \lambda^{*}$ be as in Definition 5.10. Suppose that $\mathcal{F}=\left\{\left(\mathcal{W}_{i}, L_{i, j}\right.\right.$, $\left.\left.f_{i}, \mathcal{T}_{i}, \pi_{i, i+1}, \psi_{i, i+1}\right) \mid i \in \mathbb{N}, j \geq 0\right\}$ is a determining system for $\mathcal{C}$ with filtering $\mathcal{F}_{0}=\left\{\left(\mathcal{R}_{i}, \lambda_{i}, \psi_{i}\right) \mid i \in \mathbb{N}\right\}$ for $\mathcal{F}$ subject to the conditions that for each $i \in \mathbb{N}, \mathcal{W}_{i}=\lambda_{\mathcal{C}}\left(\mathcal{R}_{i}\right)$ and $\lambda_{i}=\lambda_{f_{i}}^{*}$. Then there exists a determining system $\mathcal{G}=\left\{\left(\mathcal{U}_{i}, K_{i, j}, g_{i}, \widetilde{\mathcal{T}}_{i}, \widetilde{\pi}_{i, i+1}, \widetilde{\psi}_{i, i+1}\right) \mid i \in \mathbb{N}, j \geq 0\right\}$ for $\mathcal{D}$ along with a filtering $\mathcal{G}_{0}=\left\{\left(\mathcal{S}_{i}, \widetilde{\lambda}_{i}, \widetilde{\psi}_{i}\right) \mid i \in \mathbb{N}\right\}$ for $\mathcal{G}$ such that for each $i \in \mathbb{N}$,

(1) $\mathcal{U}_{i}=\lambda_{\mathcal{D}}\left(\mathcal{S}_{i}\right)$

(2) $\widetilde{\lambda}_{i}=\lambda_{g_{i}}^{*}$,

(3) $L_{i, j}$ is a subcomplex of $K_{i, j}, j=0,1, \ldots$,

(4) $\tilde{\pi}_{i, i+1} \mid L_{i+1,0}=\pi_{i, i+1}: L_{i+1,0} \rightarrow L_{i, 1} \subset K_{i, 1}$, and

(5) $\left.g_{i}\left|X=f_{i}: X \rightarrow\right| L_{i, 0}\right|_{C W} \subset\left|K_{i, 0}\right|_{C W}$. 
Pr o of. This will be obtained via a construction by induction on $\mathbb{N}$. For $n=1$, proceed as follows. Using Lemma 4.10, we find a progressive expansion $\mu_{1}: \mathcal{R}_{1} \rightarrow \mathcal{S}_{1}$ of $\mathcal{R}_{1}$ in $Y$ in such a manner that $\mathcal{S}_{1}$ refines $\operatorname{Cov}\left(Y, 1 / 2^{2}\right)$ and $\mathcal{S}_{1}$ is a locally finite open cover of $Y$. According to Definition 5.10, there is a progressive expansion $\theta_{1}: \mathcal{W}_{1} \rightarrow \mathcal{U}_{1}=\mathcal{U}\left(\mathcal{S}_{1}\right)$ in $Y$ such that for each $W \in \mathcal{W}_{1}, \theta_{1}(W) \subset \mu_{1} \circ \lambda_{1}(W)$, and there is a $\mathcal{U}_{1}$-normal map $g_{1}: Y \rightarrow\left|K_{1,0}\right|_{C W}$ such that $\left.g_{1}\left|X=f_{1}: X \rightarrow\right| L_{1,0}\right|_{C W} \subset\left|K_{1,0}\right|_{C W}$, where $K_{1,0}=N\left(\mathcal{U}_{1}\right)$. Here we treat $L_{1,0}$ as a subcomplex of $K_{1,0}$ via $\theta_{1}$.

Recursively extend each of the subdivisions $L_{i, j}$ of $\beta_{2} L_{1, j-1}$ to a subdivision $K_{i, j}$ of $\beta_{2} K_{1, j-1}, j \in \mathbb{N}$. Choose $\widetilde{\lambda}_{1}=\lambda_{g_{1}}^{*}$.

As far as the determining system $\mathcal{G}$ and its associated filtering are concerned, we have constructed all applicable objects for $n=1$. Namely, the elements of $\left\{\left(\mathcal{U}_{1}, K_{1, j}, g_{1}, \widetilde{\mathcal{T}}_{1}\right) \mid j \geq 0\right\}$ satisfy (1)-(3) of Lemma 7.1 and $\left(\mathcal{S}_{1}, \widetilde{\lambda}_{1}\right)$ satisfies $(1)-(3)$ of Definition 7.2. Also, our construction meets the requirements of (1)-(3) and (5) of the current lemma. Everything else is true vacuously.

Proceeding inductively, assume that $n \in \mathbb{N}$ and for each $1<i \leq n$ we have constructed $\left\{\left(U_{i}, K_{i, j}, g_{i}, \widetilde{\mathcal{T}}_{i}, \widetilde{\pi}_{i, i+1}, \widetilde{\psi}_{i, i+1}\right) \mid j \geq 0\right\}$ and $\left(\mathcal{S}_{i}, \widetilde{\lambda}_{i}, \widetilde{\psi}_{i}\right)$ so that (1)-(7) of Lemma 7.1, (1)-(5) of Definition 7.2, and (1)-(5) of the hypothesis hold true for all applicable indexes. We want to produce the data for index $n+1$.

Consider the finite collection of open covers of $Y$ consisting of $\operatorname{Cov}\left(Y, 1 / 2^{n+3}\right)$ and all $g_{s}^{-1}\left(\right.$ st $\left.K_{s, k}\right), s<n+1$ and $k \leq n+2$. By (3) of $7.2, \mathcal{R}_{n+1}$ refines $\operatorname{Cov}\left(X, 1 / 2^{n+3}\right)=\operatorname{Cov}\left(Y, 1 / 2^{n+3}\right) \mid X$, therefore it refines $\operatorname{Cov}\left(Y, 1 / 2^{n+3}\right)$. Similarly, but this time using (4) of 7.2 and (5) of the hypothesis, one sees that $\mathcal{R}_{n+1}$ refines each of the covers $g_{s}^{-1}\left(\right.$ st $\left.K_{s, k}\right)$ just mentioned. Let $\Omega$ be the open cover of $Y$ which is the intersection of these $g_{s}^{-1}\left(\right.$ st $\left.K_{s, k}\right)$ and $\operatorname{Cov}\left(Y, 1 / 2^{n+3}\right)$.

We have $\psi_{n}: \mathcal{R}_{n+1} \rightarrow f_{n}^{-1}\left(\right.$ st $\left.L_{n, 1}\right)$ from $7.2(5)$; let us denote $\psi_{n}(R)$ as $f_{n}^{-1} \operatorname{st}\left(v_{R}, L_{n, 1}\right) \subset g_{n}^{-1} \operatorname{st}\left(v_{R}, K_{n, 1}\right), R \in \mathcal{R}_{n+1}$. Since $\psi_{n}$ is a projection, we have $R \subset \psi_{n}(R) \subset g_{n}^{-1} \operatorname{st}\left(v_{R}, K_{n, 1}\right)$. Choose an element $\lambda^{\prime}(R) \in \Omega$ such that $R \subset \lambda^{\prime}(R) \subset g_{n}^{-1} \operatorname{st}\left(v_{R}, K_{n, 1}\right)$. We then apply Lemma 4.10 to get a locally finite open cover $\mathcal{S}_{n+1}$ of $Y$ and a progressive expansion $\mu: \mathcal{R}_{n+1} \rightarrow \mathcal{S}_{n+1}$ of $\mathcal{R}_{n+1}$ in $Y$ such that $R \subset \mu(R) \subset \lambda^{\prime}(R)$ for each $R \in \mathcal{R}_{n+1}$. Moreover, we choose $\mathcal{S}_{n+1}$ so that $\mathcal{S}_{n+1}$ refines $\Omega$.

Define $\widetilde{\psi}_{n}: \mathcal{S}_{n+1} \rightarrow g_{n}^{-1}$ (st $\left.K_{n, 1}\right)$ in the following way. If $S=\mu(R) \in$ $\mu\left(\mathcal{R}_{n+1}\right) \subset \mathcal{S}_{n+1}$, then $S \subset \lambda^{\prime}(R) \subset g_{n}^{-1} \operatorname{st}\left(v_{R}, K_{n, 1}\right)$, and we let $\widetilde{\psi}_{n}(S)=$ $g_{n}^{-1} \operatorname{st}\left(v_{R}, K_{n, 1}\right)$. So we infer that $\widetilde{\psi}_{n}(\mu(R))=g_{n}^{-1} \operatorname{st}\left(v_{R}, K_{n, 1}\right)$ if and only if $\psi_{n}(R)=f_{n}^{-1} \operatorname{st}\left(v_{R}, L_{n, 1}\right)$. For an element $S$ of $\mathcal{S}_{n+1} \backslash \mu\left(\mathcal{R}_{n+1}\right)$, just choose arbitrarily an element $\widetilde{\psi}_{n}(S)$ of $g_{n}^{-1}\left(\right.$ st $\left.K_{n, 1}\right)$ which contains $S$. Thus, $\widetilde{\psi}_{n}$ : $\mathcal{S}_{n+1} \rightarrow g_{n}^{-1}\left(\right.$ st $\left.K_{n, 1}\right)$ is a projection. 
One can see that as far as this choice of $\mathcal{S}_{n+1}$, we have certainly satisfied the conditions (1), (3), (4) of Definition 7.2. Applying Definition 5.10, we choose $\mathcal{U}_{n+1}=\lambda_{\mathcal{D}}\left(\mathcal{S}_{n+1}\right)$ and a $\mathcal{U}_{n+1}$-normal map $g_{n+1}: Y \rightarrow\left|K_{n+1,0}\right|_{C W}$ such that $\left.g_{n+1}\left|X=f_{n+1}: X \rightarrow\right| L_{n+1,0}\right|_{C W} \subset\left|K_{n+1,0}\right|_{C W}$, where of course $K_{n+1,0}$ is the nerve of $\mathcal{U}_{n+1}$. Also keep in mind that we use the progressive expansion $\theta=\theta_{n+1}: \mathcal{W}_{n+1} \rightarrow \mathcal{U}_{n+1}$ of 5.10 to treat $L_{n+1,0}$ as a subcomplex of $K_{n+1,0}$.

Since $\mathcal{U}_{n+1}$ refines $\mathcal{S}_{n+1}$ which refines $\Omega$, (2) of 7.2 and (1), (4) and (7) of 7.1 are true. Obviously, $7.1(2)$ is satisfied. Setting $\widetilde{\lambda}_{n+1}=\lambda_{g_{n+1}}^{*}: \mathcal{U}_{n+1} \rightarrow$ $\mathcal{S}_{n+1}$, one sees that (1), (2) and (5) of this lemma are also satisfied.

Next let us define $\widetilde{\psi}_{n, n+1}: \mathcal{U}_{n+1} \rightarrow g_{n}^{-1}\left(\right.$ st $\left.K_{n, 1}\right)$ to be the composition $\widetilde{\psi}_{n} \circ \widetilde{\lambda}_{n+1}$. Thus $\widetilde{\psi}_{n, n+1}$ is a projection. By means of this projection, we induce the simplicial map $\tilde{\pi}_{n, n+1}: K_{n+1,0} \rightarrow K_{n, 1}$, thus simultaneously obtaining 7.2(5) and 7.1(5). Hence all parts of 7.2 have been checked with these new data.

We want to ascertain the truth of (4) of this lemma. Consider a vertex $W$ of $L_{n+1,0}$, i.e., $W \in \mathcal{W}_{n+1}$. Then $\pi_{n, n+1}(W)$ is the unique vertex $x_{W}$ of $L_{n, 1}$ such that $\psi_{n, n+1}(W)=f_{n}^{-1} \operatorname{st}\left(x_{W}, L_{n, 1}\right)$. We need to show that $\tilde{\pi}_{n, n+1}(W)=x_{W}$. Note that by (5) of 7.2 , we have $f_{n}^{-1} \operatorname{st}\left(x_{W}, L_{n, 1}\right)=$ $\psi_{n, n+1}(W)=\psi_{n} \circ \lambda_{n+1}(W)=\psi_{n} \circ \lambda_{f_{n+1}}^{*}(W)$.

When we treat $L_{n+1,0}$ as a subcomplex of $K_{n+1,0}$, we do so via the progressive expansion $\theta_{n+1}: \mathcal{W}_{n+1} \rightarrow \mathcal{U}_{n+1}$ of 5.10 , where $\mu \circ \lambda_{f_{n+1}}^{*}(W)=$ $\lambda_{g_{n+1}}^{*} \circ \theta_{n+1}(W), W \in \mathcal{W}_{n+1}$. Hence showing $\widetilde{\pi}_{n, n+1}(W)=x_{W}$ really means showing that $\widetilde{\pi}_{n, n+1}\left(\theta_{n+1}(W)\right)=x_{W}$. This is equivalent to proving that $\widetilde{\psi}_{n, n+1}\left(\theta_{n+1}(W)\right)=g_{n}^{-1} \operatorname{st}\left(x_{W}, K_{n, 1}\right)$.

One has $\widetilde{\psi}_{n, n+1}\left(\theta_{n+1}(W)\right)=\widetilde{\psi}_{n} \circ \widetilde{\lambda}_{n+1}(\theta(W))=\widetilde{\psi}_{n} \circ \lambda_{g_{n+1}}^{*}(\theta(W))=$ $\widetilde{\psi}_{n} \circ \mu \circ \lambda_{f_{n+1}}^{*}(W)$. Let $S=\mu \circ \lambda_{f_{n+1}}^{*}(W)$ and $R=\lambda_{f_{n+1}}^{*}(W)$, and apply the definition of $\widetilde{\psi}_{n}$. We have $\widetilde{\psi}_{n}(S)=g_{n}^{-1} \operatorname{st}\left(v_{R}, K_{n, 1}\right)$, where $\psi_{n}(R)=$ $f_{n}^{-1} \operatorname{st}\left(v_{R}, L_{n, 1}\right)$. Thus $\widetilde{\psi}_{n, n+1}\left(\theta_{n+1}(W)\right)=g_{n}^{-1} \operatorname{st}\left(v_{R}, K_{n, 1}\right)$. We need to show that $v_{R}=x_{W}$. But from a previous determination, $\psi_{n}(R)=\psi_{n} \circ$ $\lambda_{f_{n+1}}^{*}(W)=f_{n}^{-1} \operatorname{st}\left(x_{W}, L_{n, 1}\right)$. If $v_{R} \neq x_{W}$, then $\operatorname{st}\left(v_{R}, L_{n, 1}\right) \neq \operatorname{st}\left(x_{W}, L_{n, 1}\right)$. And since $f_{n}$ is a surjection, then $f_{n}^{-1} \operatorname{st}\left(v_{R}, L_{n, 1}\right) \neq f_{n}^{-1} \operatorname{st}\left(x_{W}, L_{n, 1}\right)$, which shows that $\psi_{n}(R) \neq \psi_{n}(R)$, a contradiction. Hence we have proved (4).

In order to get (3) of the current lemma, it is necessary to select complexes $K_{n+1, j}, j=1,2, \ldots$, so that $L_{n+1, j}$ is a subcomplex of $K_{n+1, j}$. On the other hand, we must also do this so that (3), (6) of 7.1 are true relative to the complexes of type $K_{i, j}$. The procedure we use is recursive.

Consider the open cover $\widetilde{\pi}_{n, n+1}^{-1}\left(\right.$ st $\left.K_{n, n+1}\right)$ of $\left|K_{n+1,0}\right|_{C W}$ (the function $\widetilde{\pi}_{n, n+1}$ is simplicial and hence is continuous in $\left.C W\right)$. Use Theorem 3.7 to find a subdivision $K_{n+1,1}$ of $K_{n+1,0}$ which refines $\beta_{2} K_{n+1,0}$, extends the 
subdivision $L_{n+1,1}$ of $L_{n+1,0}$, and has the property that $\overline{\text { st }} K_{n+1,1}$ refines $\widetilde{\pi}_{n, n+1}^{-1}\left(\right.$ st $\left.K_{n, n+1}\right)$. One makes use here of the fact that $L_{n+1,1}$ is a subdivision of $\beta_{2} L_{n+1,0}$ by definition of sequential star-uniformity, and that since $\pi_{n, n+1}$ is the restriction of $\widetilde{\pi}_{n, n+1}$ to $\left|L_{m+1,0}\right|$, it follows that $\overline{\mathrm{st}} L_{n+1,1}$ refines $\widetilde{\pi}_{n, n+1}^{-1}\left(\right.$ st $\left.K_{n, n+1}\right)$. This is the first step in a process which can be continued inductively to produce (3), (6) of 7.1 and (3) of this lemma.

Our proof is complete.

7.4. TheOREM. Every metric space $Y$ supports a definitive system in which the maps $f_{i}$ of Definition $6.3(2)$ are $K_{i, 0}$-irreducible.

Proof. Let $X=\mathcal{C}=\emptyset$ and let $\mathcal{D}$ be the collection of all principal refinements in $Y$. Then by 5.14 and $5.13, \mathcal{D}$ is a fine normal extension of $\mathcal{C}$ in $Y$. From Lemma 5.6, one sees that each element of $\mathcal{D}$ has locally finite-dimensional nerve.

We choose the determining system $\mathcal{F}$ for $\mathcal{C}$ in which all entries are empty. Then there is a filtering for $\mathcal{F}$ in which all entries are empty. By Lemma 7.3, there is a determining system $\mathcal{G}$ for $\mathcal{D}$. By Definition 7.2, the determining system $\mathcal{G}$ induces the data in Lemma 7.1, and so by Lemma 7.1, there exists such a definitive system $\mathcal{S}$ for $Y$.

An analysis of this proof shows even more.

7.5. TheOREM. Let $\mathcal{D}$ be a cofinal collection of strongly normal, locally finite covers of a metric space $X$, each having locally finite-dimensional nerve. Then there is a sequence $\mathcal{W}_{1}, \mathcal{W}_{2}, \ldots$ of elements of $\mathcal{D}$ so that all the data of 7.1 can be satisfied. Hence there is a definitive system $\mathcal{S}$ for $X$ where each $L_{i, 0}$ is the nerve of an element of $\mathcal{D}$, and $f_{i}: X \rightarrow\left|L_{i, 0}\right| \mathcal{T}_{i}$ is a $\mathcal{W}_{i}$-normal map.

We want to explore a further ramification of Lemma 7.3. The determining system $\mathcal{F}$ for $\mathcal{C}$ and its associated filtering $\mathcal{F}_{0}$ appear to have a certain extension property. This manifests itself in that whenever $\mathcal{D}$ is a fine normal extension of $\mathcal{C}$ in $Y$, one gets a determining system $\mathcal{G}$ for $\mathcal{D}$ along with a filtering $\mathcal{G}_{0}$ which are of the same type relative to $\mathcal{D}$ as their predecessors were relative to $\mathcal{C}$. Furthermore, there are certain extensions of maps and polyhedra which also occur. We put this now into a definition.

7.6. Definition. Let $X$ be a metric space, $\mathcal{C}$ be a collection of normal covers of $X$ each having locally finite-dimensional nerve and so that $\mathcal{C}$ is central for $X$. A pair $\left(\mathcal{F}, \mathcal{F}_{0}\right)$ will be called an extendable filtered determining system for $\mathcal{C}$ if $\mathcal{F}=\left\{\left(\mathcal{W}_{i}, L_{i, j}, f_{i}, \mathcal{T}_{i}, \pi_{i, i+1}, \psi_{i, i+1}\right) \mid i \in \mathbb{N}, j \geq 0\right\}$ is a determining system for $\mathcal{C}$ with filtering $\mathcal{F}_{0}=\left\{\left(\mathcal{R}_{i}, \lambda_{i}, \psi_{i}\right) \mid i \in \mathbb{N}\right\}$ for $\mathcal{F}$ such that for each $i \in \mathbb{N}, \mathcal{W}_{i}=\lambda_{\mathcal{C}}\left(\mathcal{R}_{i}\right)$ and $\lambda_{i}=\lambda_{f_{i}}^{*}$.

Let us apply this definition and Lemma 7.3 to a specific situation. 
7.7. Lemma. Let $X$ be a closed subspace of a metric space $Y$ and $\mathcal{C}$ be the collection of all principal refinements of $X$. Then there exists an extendable filtered determining system $\left(\mathcal{F}, \mathcal{F}_{0}\right)$ for $\mathcal{C}$. Let $\mathcal{D}$ be the collection of all principal refinements of $Y$. Then there exists an extendable filtered determining system $\left(\mathcal{G}, \mathcal{G}_{0}\right)$ for $\mathcal{D}$, dependent upon $\left(\mathcal{F}, \mathcal{F}_{0}\right)$, so that $(3)-(5)$ of Lemma 7.3 are true.

Proof. We know from Theorem 5.11 that $\mathcal{C}$ is central for $X$, and from Definition 5.7 that each element of $\mathcal{C}$ has locally finite-dimensional nerve. Our proof of Theorem 7.4 shows that there is an extendable filtered determining system $\left(\mathcal{F}, \mathcal{F}_{0}\right)$ for $\mathcal{C}$.

Now, Theorem 5.11 yields that $\mathcal{D}$ is a fine normal extension of $\mathcal{C}$ in $Y$, and of course each element of $\mathcal{D}$ has locally finite-dimensional nerve. Lemma 7.3 then produces the required pair $\left(\mathcal{G}, \mathcal{G}_{0}\right)$.

8. Representations of strongly countable-dimensional spaces. Let us recall that a normal space is called strongly countable-dimensional if it can be written as a countable union of closed, finite-dimensional subspaces. The survey article [E-P] deals with countable-dimensional spaces in general, and is a good source of information on this subject. The next result is easily seen to be true.

8.1. Lemma. Every subspace of a strongly countable-dimensional metrizable space is strongly countable-dimensional.

8.2. Definition. Let $\left(g_{i}\right)_{i=1}^{\infty}$ be a polyhedral representation of a space $X$ in an inverse sequence $\mathbf{P}=\left(P_{i}, \pi_{i, i+1}, \mathbb{N}\right)$ of metrizable polyhedra $P_{i}$ each having triangulation $K_{i}$ as in Definition 6.1. We shall call the representation strongly countable-dimensional if for each $x \in X$ we have $\sup \left\{\operatorname{dim} \sigma_{i}(x) \mid\right.$ $i \in \mathbb{N}\}<\infty$, where by $\sigma_{i}(x)$ we mean the carrier of $g_{i}(x)$ in $K_{i}$.

Let us now state our characterization of strong countable-dimensionality for metrizable spaces.

8.3. Theorem. Let $X$ be a metrizable space. Then $X$ is strongly countable-dimensional if and only if $X$ has a strongly countable-dimensional representation.

We shall need to lay some groundwork before proceeding to prove this theorem.

8.4. Lemma. If a space $X$ has a strongly countable-dimensional representation, then $X$ is a strongly countable-dimensional metrizable space.

P r o o f. Let us assume the notation of Definition 8.2. Since the polyhedra $P_{i}$ are metrizable, so is $\lim \mathbf{P}$. Hence the space $X$, which embeds in $\lim \mathbf{P}$, is metrizable. 
Now let us determine a strongly countable-dimensional subspace of $\lim \mathbf{P}$ which contains $g(X)$, where $g$ is the embedding $\prod_{i=1}^{\infty} g_{i}: X \rightarrow \lim \mathbf{P}$. If we can do this, then Lemma 8.1 will yield the desired conclusion.

Fix $n \in \mathbb{N}$. Let $Y_{1, n}=\left|K_{1}^{(n)}\right|_{\mathcal{T}_{1}}$ and inductively, for $i \in \mathbb{N}$, let $Y_{i+1, n}=$ $\pi_{i, i+1}^{-1}\left(Y_{i, n}\right) \cap\left|K_{i+1}^{(n)}\right|_{\mathcal{T}_{i+1}}$. From $3.5(\mathrm{v})$, each $\left|K_{i}^{(n)}\right|$ is closed in $P_{i}$. It follows that $Y_{n}=\lim \left(Y_{i, n}, \pi_{i, i+1} \mid Y_{i+1, n}, \mathbb{N}\right)$ is a closed subspace of $\lim \mathbf{P}$. From 3.5 (viii), $\operatorname{dim} Y_{i, n} \leq n$ for each $i$. By 27.9 of [Nam], $\operatorname{dim} Y_{n} \leq n$. Our proof will therefore be complete if we can show that $g(X) \subset \bigcup_{n=1}^{\infty} Y_{n}$.

Let $x \in X$ and let $\tau_{i} \in K_{i}$ be the carrier of $g_{i}(x), i \in \mathbb{N}$. Then for each $i \in \mathbb{N}, \operatorname{dim} \tau_{i} \leq n=\sup \left\{\operatorname{dim} \tau_{j} \mid j \in \mathbb{N}\right\}<\infty$. Clearly $g_{1}(x) \in Y_{1, n}$. Suppose inductively that $g_{k}(x) \in Y_{k, n}, 1 \leq k \leq i$. We need to show that $g_{i+1}(x) \in Y_{i+1, n}$.

Now $\pi_{i, i+1} g_{i+1}(x)=g_{i}(x) \in Y_{i, n}$. Hence $g_{i+1}(x) \in \pi_{i, i+1}^{-1}\left(Y_{i, n}\right)$. But also $g_{i+1}(x) \in\left|K_{i+1}^{(n)}\right|$. This shows that $g_{i+1}(x) \in Y_{i+1, n}$, and our lemma is proved.

This lemma, of course, proves the necessity part of Theorem 8.3. For the sufficiency we need to develop some additional ideas.

8.5. Lemma. Let $X$ be a strongly countable-dimensional metric space. Then there exists a function $n: X \rightarrow \mathbb{Z}$ such that every open cover $\mathcal{U}$ of $X$ has an open refinement $\mathcal{V}$ covering $X$ with $\operatorname{ord}_{x} \mathcal{V} \leq n(x)+1$ for each $x \in X\left(\operatorname{ord}_{x} \mathcal{V}\right.$ means the number of elements of $\mathcal{V}$ that contain $\left.x\right)$.

Proof (extracted from the proof of the necessity part of Theorem 5.3 of [Nat]). Let $X=\bigcup_{k=1}^{\infty} F_{k}$, where each $F_{k}$ is a closed subset of $X$ and $\operatorname{dim} F_{k}=n_{k}<\infty$. For each $x \in X$ let $l(x)=m(x)+\sum_{k=1}^{m(x)} n_{k}$, where $m(x)=\min \left\{k \mid x \in F_{k}\right\}$. Define $n(x)$ to be $l(x)-1$. Since $\operatorname{dim} F_{k}=n_{k}$ there is an open cover $\mathcal{U}_{k}$ of $F_{k}$ with ord $\mathcal{U}_{k} \leq n_{k}+1$ and $\mathcal{U}_{k}$ precisely refines $\mathcal{U} \cap F_{k}$. Suppose $\mathcal{U}_{k}=\left\{U_{\alpha} \mid \alpha \in \Gamma\right\}$. For each $x \in U_{\alpha}$ we find $\varepsilon(x)>0$ such that the $\varepsilon(x)$-ball $B(x, \varepsilon(x)) \subseteq S_{\alpha} \in \mathcal{U}$ and $B(x, \varepsilon(x)) \cap F_{k} \subseteq U_{\alpha}$. Set $U_{a}^{\prime}=\bigcup\left\{B(x, \varepsilon(x) / 2) \mid x \in U_{a}\right\}$ and let $\mathcal{U}_{k}^{\prime}=\left\{U_{\alpha}^{\prime} \mid \alpha \in \Gamma\right\}$. Then $\mathcal{U}_{k}^{\prime}$ refines $\mathcal{U}$ and $\operatorname{ord} \mathcal{U}_{k}^{\prime} \leq n_{k}+1$.

Now $\mathcal{V}_{k}=\left\{\left(X \backslash \bigcup_{i=1}^{k-1} F_{i}\right) \cap U_{\alpha}^{\prime} \mid \alpha \in \Gamma\right\}$ is a collection of open sets covering $F_{k} \backslash \bigcup_{i=1}^{k-1} F_{i}$ such that $\mathcal{V}_{k}$ refines $\mathcal{U}, \operatorname{ord}_{x} \mathcal{V}_{k} \leq n_{k}+1$ for $x \in \bigcup_{i=k}^{\infty} F_{i}$, and $\operatorname{ord}_{x} \mathcal{V}_{k}=0$ for $x \in \bigcup_{i=1}^{k-1} F_{i}$.

Thus $\mathcal{V}=\bigcup_{k=1}^{\infty} \mathcal{V}_{k}$ is the desired open cover of $X$.

8.6. Lemma. Let $\mathcal{U}=\left\{U_{\alpha} \mid \alpha \in A\right\}$ and $\mathcal{V}=\left\{V_{\beta} \mid \beta \in B\right\}$ be open covers of a space $X$ such that $\mathcal{V}$ refines $\mathcal{U}$. Then there exists an open cover $\mathcal{W}$ of $X$ such that $\mathcal{W}$ is a precise refinement of $\mathcal{U}$ and $\operatorname{ord}_{x} \mathcal{W} \leq \operatorname{ord}_{x} \mathcal{V}$ for each $x \in X$. 
Proof. Define a function $\varphi: B \rightarrow A$ by assigning to each $\beta \in B$ an $\alpha \in$ $A$ such that $V_{\beta} \subseteq U_{\alpha}$. For each $\alpha \in A$ let $W_{\alpha}=\bigcup\left\{V_{\beta} \mid \varphi(\beta)=\alpha\right\}$; then each $W_{\alpha}$ is open, $W_{\alpha} \subseteq U_{\alpha}$, and $X \subseteq \bigcup_{\beta \in \beta} V_{\beta}=\bigcup_{\alpha \in A} W_{\alpha}$. Hence the collection $\mathcal{W}=\left\{W_{\alpha} \mid \alpha \in A\right\}$ is a precise refinement of $\mathcal{U}$ and $\mathcal{W}$ is an open cover of $X$. Further, to each $\alpha \in A$ such that $x \in W_{\alpha}(x \in X)$ there corresponds at least one $\beta \in B$ so that $x \in V_{\beta} \subseteq W_{\alpha}$. Hence $\operatorname{ord}_{x} \mathcal{W} \leq \operatorname{ord}_{x} \mathcal{V}$ for each $x \in X$.

8.7. Lemma. Let $X$ be a strongly countable-dimensional metric space. Then there exists a function $n: X \rightarrow \mathbb{Z}$ such that for every open cover $\mathcal{U}$ of $X$ there is a locally finite open cover $\mathcal{V}$ of $X$ refining $\mathcal{U}$ having the property that the nerve of $\mathcal{V}$ is locally finite-dimensional and such that $\operatorname{ord}_{x} \mathcal{V} \leq$ $n(x)+1$ for all $x \in X$.

Proof. Let $n: X \rightarrow \mathbb{Z}$ be as guaranteed by Lemma 8.5. Let $\mathcal{W}_{1}$ be a locally finite open cover of $X$ refining $\mathcal{U}$. Apply Definition 5.3 to obtain a locally finite open cover $\mathcal{W}_{2}$ of $X$ refining $\mathcal{W}_{1}$ such that the nerve of $\mathcal{W}_{2}$ is locally finite-dimensional. Using Lemma 8.5, select an open cover $\mathcal{W}_{3}$ of $X$ refining $\mathcal{W}_{2}$ such that $\operatorname{ord}_{x} \mathcal{W}_{3} \leq n(x)+1$ for all $x \in X$. Now Lemma 8.6 guarantees the existence of an open cover $\mathcal{V}$ of $X$ such that $\mathcal{V}$ is a precise refinement of $\mathcal{W}_{2}$ and $\operatorname{ord}_{x} \mathcal{V} \leq \operatorname{ord}_{x} \mathcal{W}_{3} \leq n(x)+1$ for all $x \in X$. Note that $\mathcal{V}$ refines $\mathcal{U}$ and since $\mathcal{W}_{2}$ is locally finite with locally finite-dimensional nerve, it follows that $\mathcal{V}$, being a precise refinement of $\mathcal{W}_{2}$, is also locally finite with locally finite-dimensional nerve, as desired.

8.8. Proposition. Let $X$ be a strongly countable-dimensional metric space. Then there exists a function $n: X \rightarrow \mathbb{Z}$ such that for every open cover $\mathcal{U}$ of $X$ there is a locally finite open cover $\mathcal{V}$ of $X$ refining $\mathcal{U}$ such that $\operatorname{ord}_{x} \mathcal{V} \leq n(x)+1$ for all $x \in X$, the nerve $N$ of $\mathcal{V}$ is locally finitedimensional, and there is a $\mathcal{V}$-normal map $f: X \rightarrow|N|_{C W}$.

Proof. Let $n: X \rightarrow \mathbb{Z}$ be as in Lemma 8.7, and $\mathcal{U}$ be an open cover of $X$. Let $\mathcal{V}^{\prime}$ be a locally finite open cover of $X$ refining $\mathcal{U}$ as granted by Lemma 8.7. Hence the nerve of $\mathcal{V}^{\prime}$ is locally finite-dimensional and $\operatorname{ord}_{x} \mathcal{V}^{\prime} \leq n(x)+1$ for all $x \in X$.

By Lemma 4.13, there is a precise refinement $\mathcal{V}$ of $\mathcal{V}^{\prime}$, covering $X$, having nerve $N$ and a $\mathcal{V}$-normal map $f: X \rightarrow|N|_{C W}$. Being a precise refinement of $\mathcal{V}^{\prime}, \mathcal{V}$ surely also enjoys the property $\operatorname{ord}_{x} \mathcal{V} \leq n(x)+1$ for all $x \in X$ and $N$ is locally finite-dimensional.

Pro of of Theorem 8.3. As indicated earlier, Lemma 8.4 yields the necessity. For the sufficiency, let $n: X \rightarrow \mathbb{Z}$ be a function as in Proposition 8.8. Let $\mathcal{C}_{2}$ be the collection of all open covers $\mathcal{V}$ of $X$ having the properties that $\operatorname{ord}_{x} \mathcal{V} \leq n(x)+1$ for all $x \in X, \mathcal{V}$ is locally finite, its nerve $N$ is locally finite-dimensional, and there is a $\mathcal{V}$-normal map $f: X \rightarrow|N|_{C W}$. From Proposition 8.8, one sees that $\mathcal{C}_{2}$ is cofinal. 
By Theorem 7.5, there is a definitive system $\mathcal{S}$ for $X$ where each $K_{i, 0}$ is the nerve of an element $\mathcal{W}_{i}$ of $\mathcal{C}_{2}$ and $f_{i}: X \rightarrow P_{i}$ is a $\mathcal{W}_{i}$-normal map. Since $\operatorname{ord}_{x} \mathcal{W}_{i} \leq n(x)+1$, the carrier of $f_{i}(x)$ in $K_{i, 0}$ has dimension at most $n(x)$ for each $x \in X$ and $i \in \mathbb{N}$. An application of Lemma 6.5 shows that $g_{i}$ is a $K_{i, 0}$-modification of $f_{i}$, which in turn shows that $\operatorname{dim} \sigma_{i}(x) \leq n(x)$ for all $x \in X$ (see Definition 8.2). Putting this together with Corollary 6.6, our proof of the sufficiency is complete.

9. Extendable inverse sequences. In Section 3 of $[\mathrm{J}-\mathrm{R}]$ it was shown that every metric compactum has an extendable inverse sequence of finite polyhedra. We are going to generalize those results to the case of arbitrary metric spaces. It is necessary to provide definitions.

9.1. Definition. Let $\mathbf{P}=\left(P_{i}, \pi_{i, i+1}, \mathbb{N}\right), \mathbf{Q}=\left(Q_{i}, \theta_{i, i+1}, \mathbb{N}\right)$ be inverse sequences of metrizable polyhedra. We shall say that $\mathbf{Q}$ is an extension of $\mathbf{P}$ if for each $i \in \mathbb{N}, P_{i}$ is a closed subspace of $Q_{i}$ and $\theta_{i, i+1} \mid P_{i+1}=\pi_{i, i+1}: P_{i+1}$ $\rightarrow P_{i}$.

9.2. Definition. Let $\left(g_{i}\right)_{i=1}^{\infty}$ be a simplicially irreducible representation of a metrizable space $X$ in an inverse sequence $\mathbf{P}$, where for each $i, K_{i}$ is the given triangulation of $P_{i}$ and $\pi_{i, i+1}$ carries $K_{i+1}$ simplicially to the subdivision $L_{i}$ of $K_{i}$. We shall say that $\left(g_{i}\right)_{i=1}^{\infty}$ is extendable if whenever $X$ is a closed subspace of a metric space $Y$, then there exist $\mathbf{Q}$ such that $\mathbf{Q}$ is an extension of $\mathbf{P}$ and a simplicially irreducible representation $\left(h_{i}\right)_{i=1}^{\infty}$ of $Y$ in $\mathbf{Q}$ so that $h_{i} \mid X=g_{i}: X \rightarrow P_{i} \subset Q_{i}$ for each $i \in \mathbb{N}$, requiring also that, if $\widetilde{K}_{i}$ denotes the given triangulation of $Q_{i}$, and $\widetilde{L}_{i}$ is the subdivision of $\widetilde{K}_{i}$ such that $\theta_{i, i+1}$ is simplicial from $\widetilde{K}_{i+1}$ to $\widetilde{L}_{i}$, then $K_{i}$ is a subcomplex of $\widetilde{K}_{i}$ and $L_{i}$ is a subcomplex of $\widetilde{L}_{i}$. We shall refer to $\left(h_{i}\right)_{i=1}^{\infty}$ as an extension of $\left(g_{i}\right)_{i=1}^{\infty}$ to $Y$.

9.3. Definition. Let $\mathcal{E}$ denote the class of all extendable representations. There is a subclass $\mathcal{R E}$ of $\mathcal{E}$ which may be described as follows. Suppose $X$ is a closed subspace of a metrizable space $Y$ and $\left(g_{i}\right)_{i=1}^{\infty}$ is a representation of $X$ lying in $\mathcal{R E}$. Then we may choose an extension $\left(h_{i}\right)_{i=1}^{\infty}$ of $\left(g_{i}\right)_{i=1}^{\infty}$ to $Y$ in such a manner that $\left(h_{i}\right)_{i=1}^{\infty}$ also lies in $\mathcal{R E}$. Let us call the elements of $\mathcal{R E}$ recursively extendable. (Of course, we mean for $\mathcal{R E}$ to be the maximal class with this property, and similarly for $\mathcal{R F} \mathcal{F}$ in Definition 9.4 below.)

9.4. Definition. Let $\mathcal{F} \mathcal{E}$ denote the subclass of $\mathcal{E}$ such that if $\left(g_{i}\right)_{i=1}^{\infty}$ is in $\mathcal{F E}$ then it is a representation of a completely metrizable space $X$ and the embedding $\prod_{i=1}^{\infty} g_{i}$ is actually a homeomorphism. These will be called the faithful representations. There is a subclass $\mathcal{R F} \mathcal{F}$ of $\mathcal{F E}$ which may be described as follows. Suppose $X$ is a closed subspace of a completely metrizable space $Y$ and $\left(g_{i}\right)_{i=1}^{\infty}$ is a representation of $X$ lying in $\mathcal{R} \mathcal{F} \mathcal{E}$. 
Then we may choose an extension $\left(h_{i}\right)_{i=1}^{\infty}$ of $\left(g_{i}\right)_{i=1}^{\infty}$ to $Y$ in such a manner that $\left(h_{i}\right)_{i=1}^{\infty}$ also lies in $\mathcal{R F} \mathcal{F}$. Let us call the elements of $\mathcal{R} \mathcal{F} \mathcal{E}$ recursively faithfully extendable.

9.5. TheOREM. Every metrizable space $X$ has a recursively extendable representation. If $X$ is completely metrizable, then it has a recursively faithfully extendable representation.

Proof. Fix a metric for $X$ and choose it to be complete if $X$ is completely metrizable. Using 7.7 , take $\left(\mathcal{F}, \mathcal{F}_{0}\right)$ to be an extendable filtered determining system for $\mathcal{C}$, the collection of all principal refinements of $X$. Let us write $\mathcal{F}=\left\{\left(\mathcal{W}_{i}, L_{i, j}, f_{i}, \mathcal{T}_{i}, \pi_{i, i+1}, \psi_{i, i+1}\right) \mid i \in \mathbb{N}, j \geq 0\right\}$

Now Lemma 7.1 yields that the data in $\mathcal{F}$ satisfy the requirements of a definitive system $\mathcal{S}_{X}$. For each $i \in \mathbb{N}$, let $g_{i}=\lim _{j \rightarrow \infty}\left(\pi_{i, j} \circ f_{j}\right)$ be as in Lemma 6.5. Then $\left(g_{i}\right)_{i=1}^{\infty}$ is a representation of $X$ in $\lim \mathbf{P}$, where $\mathbf{P}$ is the inverse sequence induced by $\mathcal{S}_{X}$. In fact, by Corollary 6.6 , this representation is simplicially irreducible and is faithful if the metric for $X$ is complete.

Now suppose $X$ is a closed subspace of a metrizable space $Y$. Extend the given metric on $X$ to one for $Y$. In the case that $X$ has been given a complete metric and $Y$ supports a complete metric, make the extended metric for $Y$ also complete (see $[\mathrm{Ba}]$ ). Let $\mathcal{D}$ be the collection of all principal refinements of $Y$, and use Lemma 7.7 to choose an extendable filtered determining system $\left(\mathcal{G}, \mathcal{G}_{0}\right)$ for $\mathcal{D}$ so that $(3)-(5)$ of Lemma 7.3 are true.

Let us write $\mathcal{G}=\left\{\left(\mathcal{U}_{i}, K_{i, j}, \widetilde{f}_{i}, \widetilde{\mathcal{T}}_{i}, \widetilde{\pi}_{i, i+1}, \widetilde{\psi}_{i, i+1}\right) \mid i \in \mathbb{N}, j \geq 0\right\}$. Then all appropriate maps, topologies and triangulations for $\mathcal{G}$ are extensions of those for $\mathcal{F}$. For example, then, if we define $\widetilde{g}_{i}=\lim _{j \rightarrow \infty}\left(\widetilde{\pi}_{i j} \circ \widetilde{f}_{j}\right)$, then we get $\widetilde{g}_{i} \mid X=g_{i}$. Since $\left(\mathcal{G}, \mathcal{G}_{0}\right)$ has the same properties as $\left(\mathcal{F}, \mathcal{F}_{0}\right)$, one could carry this procedure out indefinitely. Hence $\left(g_{i}\right)_{i=1}^{\infty}$ lies in $\mathcal{R E}$, i.e., it is recursively extendable.

Finally, if the metric for $Y$ were complete, then of course $\left(\widetilde{g}_{i}\right)_{i=1}^{\infty}$ would be faithful, and we could carry this procedure out indefinitely upon extension to complete metrics. Therefore, in this instance one sees that $\left(g_{i}\right)_{i=1}^{\infty}$ lies in $\mathcal{R} \mathcal{F} \mathcal{E}$, meaning that $\left(g_{i}\right)_{i=1}^{\infty}$ is recursively faithfully extendable.

\section{References}

[Ba] P. Bacon, Extending a complete metric, Amer. Math. Monthly 75 (1968), 642643.

[Do] C. H. Dowker, Mapping theorems for non-compact spaces, Amer. J. Math. 69 (1947), 200-242.

[E-P] R. Engelking and E. Pol, Countable-dimensional spaces: a survey, Dissertationes Math. 216 (1983).

[Fr] H. Freudenthal, Entwicklungen von Räumen und ihren Gruppen, Compositio Math. 4 (1937), 145-234. 
[Hu] S. T. Hu, Theory of Retracts, Wayne State Univ. Press, Detroit, Michigan, 1965.

[Is1] J. R. Is bell, Irreducible polyhedral expansions, Indag. Math. 64 (1961), 242-248.

[Is2] —, Uniform neighborhood retracts, Pacific J. Math. 11 (1961), 609-648.

[J-R] R. Jimenez and L. Rubin, An addition theorem for $n$-fundamental dimension in metric compacta, Topology Appl. 62 (1995), 281-297.

[Ko1] I. M. Kozlovskiǔ, On polyhedral representations of metric spaces, Dokl. Akad. Nauk SSSR 191 (1970), 985-988 (in Russian); English transl.: Soviet Math. Dokl. 11 (1970).

[Ko2] -, Absolute polyhedral expansions of metric spaces, Trans. Moscow Math. Soc. 2 (1981), 79-114; Russian original: Trudy Moskov. Mat. Obshch. 40 (1979), 83-119.

[M-S] S. Mardešić and J. Segal, Shape Theory, North-Holland, Amsterdam, 1982.

[M-U] S. Mardešić and N. Uglešić, On irreducible mappings into polyhedra, Topology Appl. 61 (1995), 187-203.

[Nam] K. Nagami, Dimension Theory, Academic Press, New York, 1970.

[Nat] J. Nagata, On the countable sum of zero-dimensional metric spaces, Fund. Math. 48 (1960), 1-14.

$[\mathrm{Ru}] \quad \mathrm{L} . \mathrm{Rubin}$, Irreducible representations of normal spaces, Proc. Amer. Math. Soc. 107 (1989), 277-283.

[R-S] L. Rubin and P. Schapiro, Cell-like maps onto non-compact spaces of finite cohomological dimension, Topology Appl. 27 (1987), 221-244.

[Sp] E. Spanier, Algebraic Topology, McGraw-Hill, New York, 1966.

[Wi] S. Willard, General Topology, Addison-Wesley, Reading, Mass., 1968.

DEPARTMENT OF MATHEMATICS

UNIVERSITY OF NORTH DAKOTA

GRAND FORKS, NORTH DAKOTA 58202

U.S.A.

E-mail: MILLSPAU@PLAINS.NODAK.EDU

DEPARTMENT OF MATHEMATICS

LANGSTON UNIVERSITY

LANGSTON, OKLAHOMA 73050

U.S.A.

E-mail: SCHAPIRO@CHAOS.LUNET.EDU

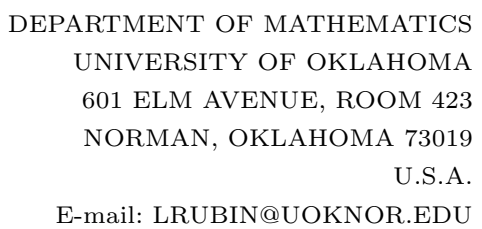

E-mail: LRUBIN@UOKNOR.EDU

Received 28 April 1994;

in revised form 12 June 1995 